\title{
Tassements et gonflements différés dans les sols fins proches de la saturation
}

\section{BOUTONNIER}

SCETAUROUTE Département Géotechnique et Matériaux

3, rue du Docteur-Schweitzer 38180 Seyssins I.boutonnier@scetauroute.fr
Des progrès remarquables ont été accomplis ces dernières années dans le domaine des sols non saturés. Cependant, il n'existe pas à l'heure actuelle de couplage hydromécanique simple permettant de prévoir les tassements et surtout les gonflements différés dans le domaine des sols fins proches de la saturation.

En utilisant la loi d'Henry, la loi des gaz parfaits, le principe des contraintes effectives et la relation $S_{r}(s)\left(S_{\text {, }}\right.$ degré de saturation, s succion), une méthode simple est proposée pour prévoir le gonflement différé sous imbibition et le tassement différé lors de l'application d'une charge (consolidation). Cette théorie monodimensionnelle peut être utilisée pour les sols contenant des bulles d'air occlus et pour des succions inférieures à la succion d'entrée d'air. Elle peut être généralisée au cas tridímensionnel.

En utilisant cette approche, une relation théorique a été établie entre le coefficient de consolidation $\mathrm{c}_{\mathrm{v} t}$ obtenu à partir des essais cedométriques classiques (pas de succion dans l'échantillon, pressions interstitielles positives) et le coefficient $c_{v 2}$ calculé à partir des courbes de gonflement en fonction du temps d'essais de gonflement libre à l'cedomètre (imbibition d'une éprouvette présentant une succion initiale). Cette relation a permis d'effectuer une étude paramétrique qui montre que, pour les sols présentant une forte succion d'entrée d'air, les coefficients $c_{v 1}$ et $c_{v 2}$ sont pratiquement égaux.

Mots-clés : tassement, gonflement, sols non saturés, consolidation.

\section{Deferred settlement and swelling forecast in fine soils near saturation}

These last decades, numerous advances have been made in unsaturated soil mechanics. However, there is currently no simple hydromechanical model to forecast the settlement or swelling as a function of time.

Using Henry's law, perfect gas law, effective stress principle and the relation $S$, (s) (S, degree of saturation, $\mathrm{S}$ suction), a simple method is proposed to predict the time evolution of swelling and settlement (consolidation). This one dimensional theory is valid for soils containing occluded air bubbles and/or for suctions below air entry suction. It could be generalised to three dimensional theory.

The model shows that the coefficient of consolidation $c_{\mathrm{v} 1}$ obtained from classical oedometric test (no suction in the sample, positive pore water pressure) and the coefficient $\mathrm{c}_{\mathrm{v}}$ obtained from « free-swell n oedometer test (submerging a specimen with initial suction) are linked. A parametric study has been carried out and it shows that $c_{y}$ and $c_{p}$ are approximately equal, particularly for solls with high air entry suction.

Key words : settlement, swelling, unsaturated soils, consolidation. 


\section{Symboles Désignations}

$a_{\text {int }}$

an

$\mathrm{C}_{\mathrm{c}}$

$\mathrm{C}_{\mathrm{ser}}$

$C_{x}$

$\mathrm{C}_{\mathrm{v}, \mathrm{m} 2}$

$C_{\text {v DIDA }}$

$\Delta e_{\text {c }}$

$\Delta e_{f}$

$\Delta u_{w i}$

$\Delta \mathrm{z}$

$\Delta \sigma$

$\Delta \sigma^{\prime}$

e

$e_{0}$

E

$\gamma_{i x}$

h

k

n

P

S

5

$S$.

$S_{\text {rait }}$

$S_{r}^{r+}$

$t$

t

$\sigma_{p}^{\prime}$

G.

o

$\sigma^{\prime}$

T

$u_{n}$

$\mathrm{u}_{\mathrm{u} \text { at? }}$

$\mathrm{u}_{\mathrm{w}}$

$u_{w}^{w}$

v

V.

$v^{3}$

$V^{\text {al }}$

$V_{\text {v }}$
Coefficient de compressibilité du fluide interstitiel dans le domaine D3

Coefficient de compressibilité de l'eau

Indice de compression

Indice de fluage

Indice de gonflement

Indice de gonflement ou coefficient de compression

suivant que le sol est surconsolidé ou normalement consolidé

Coefficient de consolidation verticale dans le domaine D2

Coefficient de consolidation verticale dans les domaines

D3 et D4

Variation d'indice des vides lors de l'application d'un

incrément de contrainte effective $\Delta \sigma$

Variation d'indice des vides liée au fluage pendant un

incrément de temps $\Delta \mathrm{t}$

Surpression interstitielle

Hauteur de la sous-couche de sol considérée

Incrément de contrainte totale

Incrément de contrainte effective

Indice des vides

Indice des vides initial sous $\sigma^{\prime}$

Module cedométrique tangent

Poids volumique de l'eau

Constante de la loi d'Henry

Perméabilité verticale

Porosité

Pression atmosphérique de l'air

Succion

Succion d'entrée d'air ou d'expulsion d'air

Deqré de saturation

Degré de saturation au point d'entrée ou d'expulsion d'air

Degré de saturation lorsque $\mathrm{s}=\mathrm{C}$

Temps

Temps fictif permettant de calculer la vitesse de fluage

Contrainte de préconsolidation

Contrainte totale verticale

Contrainte effective verticale

Contrainte effective verticale initiale en place

Température du fluide interstitiel

Pression de l'air du fluide interstitiel

Pression de l'air correspondant à la succion d'entrée d'air

Pression de l'eau du fluide interstitiel

Pression de l'eau du fluide interstitiel pour laquelle $\mathrm{S}_{+}=1$

Vitesse d'écoulement vertical (positif vers le bas)

Volume d'air total au sein du fluide interstitiel

Volume d'air dissous

Volume d'air libre

Volume total de l'élément de sol considéré

Volume des vides de l'élément de sol considéré

Volume d'eau contenu par l'élément de sol considéré

Tassement de la couche de sol considérée

Profondeur de l'élément de sol considéré

\section{Unités Commentaires}

$\mathrm{Pa}^{-1}$

$\mathrm{Pa}^{-1}$

sans

sans

sans

sans

$\mathrm{m}^{2} / \mathrm{s}$

$\mathrm{m}^{2 / 2} \mathrm{~s}$

$\mathrm{Pa}$

sans

$\mathrm{Pa}$

M

$\mathrm{Pa}$

$\mathrm{Pa}$

sans

sans

$\mathrm{Pa}$

$\mathrm{kN} / \mathrm{m}^{5}$

sans

$\mathrm{m} / \mathrm{s}$

sans

$\mathrm{Pa}$

$\mathrm{Pa}$

$\mathrm{Pa}$

sans

sans

sans

jours

$\mathrm{Pa}$

$\mathrm{Pa}$

$\mathrm{Pa}$

$\mathrm{Pa}$

${ }^{\circ} \mathrm{K}$

$\mathrm{Pa}$

$\mathrm{Pa}$

$\mathrm{Pa}$

$\mathrm{Pa}$

$\mathrm{m} / \mathrm{s}$

$\mathrm{m}^{3}$

$m^{3}$

$m^{3}$

$m^{3}$

$\mathrm{m}^{3}$

$\mathrm{m}^{3}$

$\mathrm{m}$

$\mathrm{m}$ compression du sol normalement consolidé

compression du sol sur consolidé

$V_{\text {id }}=V_{w}, h$

$\mathrm{s}=\mathrm{u}_{\mathrm{u}}-\mathrm{u}_{\mathrm{w}}$

t. est souvent appelé t ${ }^{*}$

$\sigma_{\mathrm{v}}^{\prime}=\sigma_{\mathrm{v}}-\mathrm{u}$

Par convention, ces pressions sont nulles sous la pression atmosphérique $\mathrm{P}_{\mathrm{a}}$ Par convention, ces pressions sont nulles sous la pression atmosphérique $\mathrm{P}$

$V_{\mathrm{a}}=\mathrm{V}_{\mathrm{ad}}+\mathrm{V}_{\mathrm{a}}$

$V_{v}=V_{w w}+V_{p}$

z est compté positif vers le bas 


\section{1}

\section{Introduction}

La mécanique des sols non saturés s'est beaucoup développée ces dernières années. Ces progrès sont représentês par exemple par les travaux de Alonso et al. (1990) dans la définition de lois de comportement en variables indépendantes. Les variables mises en relation sont la contrainte isotrope p, le déviateur q, la succion s et l'indice des vides e. Ces lois de comportement ont la particularité de considérer les contraintes totales plutôt que les contraintes effectives comme en mécanique des sols saturés.

Cependant, dans le domaine des sols fins proches de la saturation (succion inférieure à la succion d'entrée d'air), Biarez et al. (1988) ont montré que le principe des contraintes effectives peut s'appliquer sur des chemins de contraintes cdométriques ou isotropes.

Modaressi et al. (1996), Khalili et Khabbaz (1998), Khalili (2000), Geiser (2000), Loret et Khalili (2002) ont ensuite généralisé ces travaux (chemins de contraintes de cisaillement au triaxial, succions supérieures à la succion d'entrée d'air), ce qui permet dès à présent de faciliter grandement la modélisation des sols non saturés, les paramètres mécaniques étant les mêmes dans les domaines saturé et non saturé. Toutefois, la démonstration pour les sols non saturés du principe des contraintes effectives à l'échelle granulaire est beaucoup moins aisée que dans le domaine saturé et pose un certain nombre de questions sur ses fondements théoriques (Delage, 1987).

Toutes ces recherches, bien que très intéressantes, sont encore peu utilisées dans la pratique des bureaux d'études où les applications sont potentiellement nombreuses: remblais en sols fins, sols fins naturels non saturés posant des problèmes de tassements ou de gonflements...

Dans le cas particulier des calculs de temps de consolidation (tassements ou gonflements différés) pour des sols fins proches de la saturation (succion inférieure à la succion d'entrée d'air), il n'existe pas à notre connaissance de modèle prédictif facilement utilisable:

1) Les théories de Fredlund et Hasan (1979), Lloret et Alonso (1980) pour la consolidation des sols non saturés considèrent que les phases liquide et gazeuse sont continues ce qui n'est pas vrai pour les succions inférieures à la succion d'entrée d'air (air occlus avant la succion d'entrée d'air) ;

2) Khalili et Khabbaz (1995) utilisent la loi de Fick pour les écoulements de gaz et la loi de Darcy pour les écoulements d'eau. Loret et Khalili (2000) complètent cette approche en introduisant explicitement la perméabilité à l'air lorsque la succion est supérieure à la succion d'entrée d'air. Cependant, ces approches négligent la dissolution de l'air dans l'eau (loi d'Henry);

3) Barden (1965), Chang et Duncan (1983) proposent des théories pour les sols présentant des bulles d'air occlus mais elles ne sont pas valables quand la succion n'est plus négligeable.

En utilisant la loi de Henry, la loi des gaz parfaits, le principe des contraintes effectives et la relation $S_{r}(s)\left(S_{i}\right.$ degré de saturation, s succion), une méthode simple est proposée dans cet article pour prévoir le gonflement différé sous imbibition et le tassement différé lors de l'application d'une charge (consolidation). Cette théo- rie monodimensionnelle peut être utilisée pour les sols contenant des bulles d'air occlus et pour des succions inférieures à la succion d'entrée d'air. Elle pourrait être généralisée au cas tridimensionnel.

En utilisant cette approche, une relation théorique est ensuite établie entre le coefficient de consolidation $\mathrm{C}_{\mathrm{v}}$ obtenu à partir des essais cedométriques classiques (pas de succion dans l'échantillon, pressions interstitielles positives) et le coefficient $c_{v 2}$ calculé à partir des courbes de gonflement en fonction du temps d'essais de gonflement libre à l'cedomètre (imbibition d'une éprouvette présentant une succion initiale). Cette relation permet d'effectuer une étude paramétrique qui montre que, pour les sols présentant une forte succion d'entrée d'air, les coefficients $c_{v 1}$ et $c_{v 2}$ sont pratiquement égaux. Quelques essais, qui tendent à valider cette théorie, sont ensuite présentés.

\section{2}

\section{Présentation des hypothèses et résultats théoriques}

Les différentes notations utilisées dans la suite de cet article sont présentées dans le tableau I.

\section{1}

\section{Les différents domaines d'un sol non saturé}

Dans le cadre de cet article, l'état d'un sol est décomposé en quatre domaines en fonction du degré de saturation, comme illustré sur la figure 1. Cette décomposition a déjà été présentée par Boutonnier et Virollet (2003). Nous la rappelons, avec un certain nombre de compléments, car elle est nécessaire à la compréhension de la suite de l'article.

Par convention, $\mathrm{u}_{3}$ et $\mathrm{u}_{\mathrm{w}}$ sont les pressions d'air et eau en excès de la pression atmosphérique $\mathrm{P}_{\mathrm{a}}$.

- Domaine D1 caractérisé par $\mathrm{S}_{\text {air }} \leq \mathrm{s}$ et $\mathrm{S}_{\mathrm{s}} \leq \mathrm{S}_{\mathrm{f} \text { air }}$

La phase gazeuse est continue dans l'échantillon de sol considéré. Cet état correspond à une succion s supérieure à la succion d'entrée d'air s $\mathrm{s}_{\mathrm{wir}}$ (ou d'expulsion d'air sur un chemin d'imbibition). Cette succion s, peut être de quelques dizaines de $\mathrm{kPa}$ à plus de $1000 \mathrm{kPa}$ pour les sols argileux (Biarez et al., 1987; Fleureau et al., 1993; Zerhouni et al., 1998; Cafaro et al., 2000; Khalili, 2000; Fleureau et al, 2002).

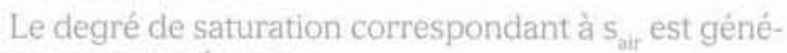
ralement compris entre:

$-S_{r \text { air }}=75 \%$ et $90 \%$ pour les sols compactés à l'optimum Proctor ou côtẻ humide (Chen et Yu, 1995, Schuurman; 1966, Chang et Duncan, 1983);

$-\mathrm{S}_{\text {rair }}=90 \%$ et $100 \%$ pour les sols initialement saturés, et en particulier les boues auxquelles on fait subir un chemin de drainage à succion croissante (Biarez et al., 1987; Fleureau et al., 1993, Zerhouni et al., 1998, Cafaro, 2000, Fleureau et al., 2002).

- Domaine D2 caractérisé par $0 \leq \mathrm{S} \leq \mathrm{S}_{\text {air }}$ et $\mathrm{S}_{r \text { air }} \leq \mathrm{S}_{r} \leq \mathrm{S}_{r E}$. L'air est occlus dans l'échantillon de sol considéré. Chaque bulle d'air est en contact avec plusieurs grains de sol et la succion $\mathrm{s}=\mathrm{u}_{\mathrm{u}}-\mathrm{u}_{\mathrm{u}}$ a pour effet d'augmenter les forces de contact intergranulaires. Par définition. 


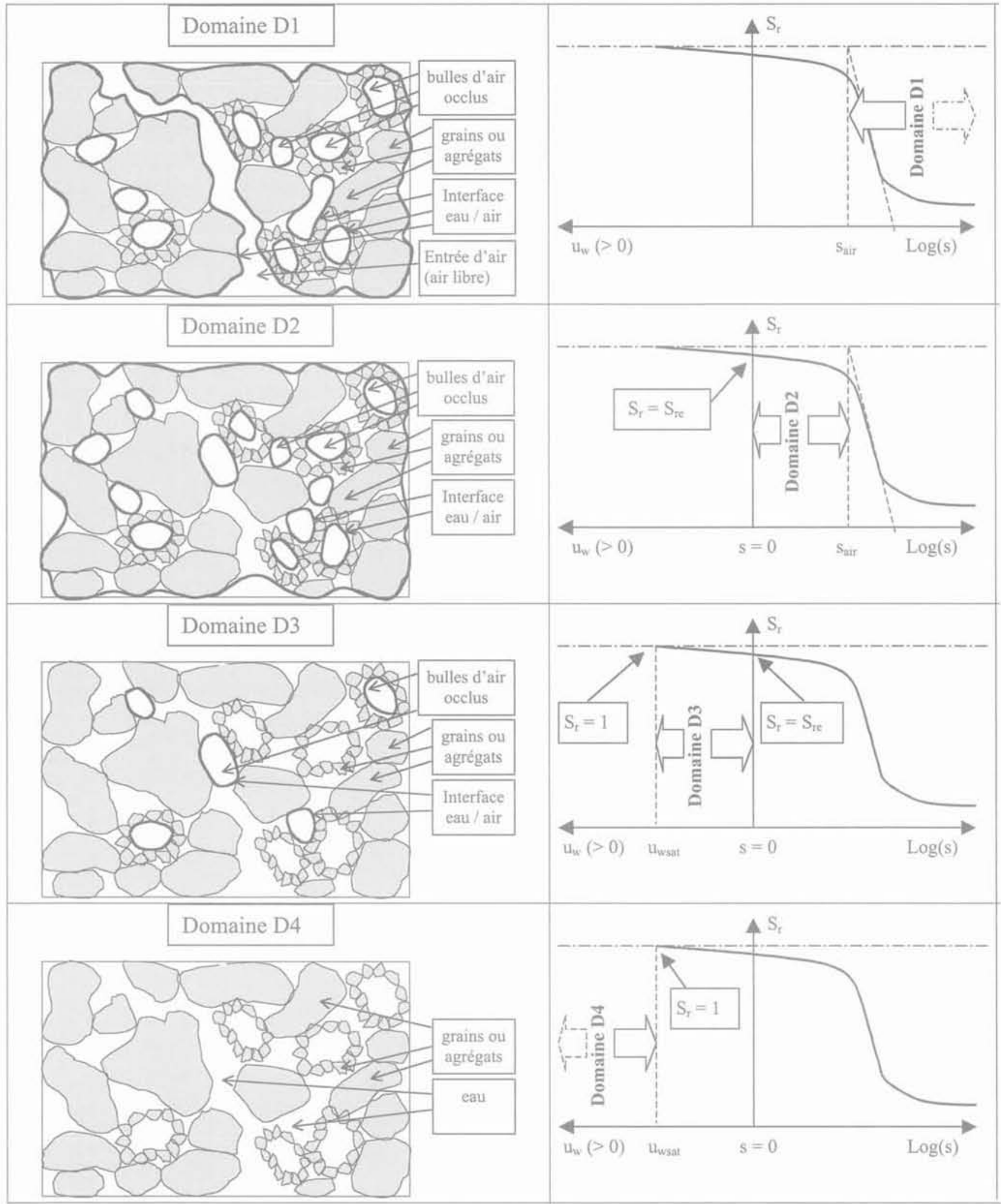

FG. 1 Représentation schématique de l'état de saturation en fonction de la succion. Schematic representation of saturation state as a function of suction.

nous appelons $\mathrm{S}_{r e r}$ le degré de saturation pour lequel la succion mesurée à l'échelle macroscopique est nulle.

Dans ce domaine, la perméabilité à l'air est nulle (la phase air n'est pas continue).

De nombreux résultats expérimentaux (Barden, 1974; Lins et al., 1995) montrent que le domaine D2 couvre l'optimum Proctor et une partie du côté humide de l'OPN (optimum Proctor normal).

- Domaine D3 caractérisé par $\mathrm{S}_{r e}<\mathrm{S}_{\mathrm{f}}<1$ et $\mathrm{S}=0$ ce qui conduit à $\mathrm{u}_{\mathrm{a}}=\mathrm{u}_{\mathrm{w}}$ avec $\mathrm{u}_{\mathrm{w}}>0$.

L'air est occlus dans l'échantillon de sol considéré. A l'échelle macroscopique, on ne mesure plus de suc- 


\section{$P_{\mathrm{n}}$ : pression atmosphérique}

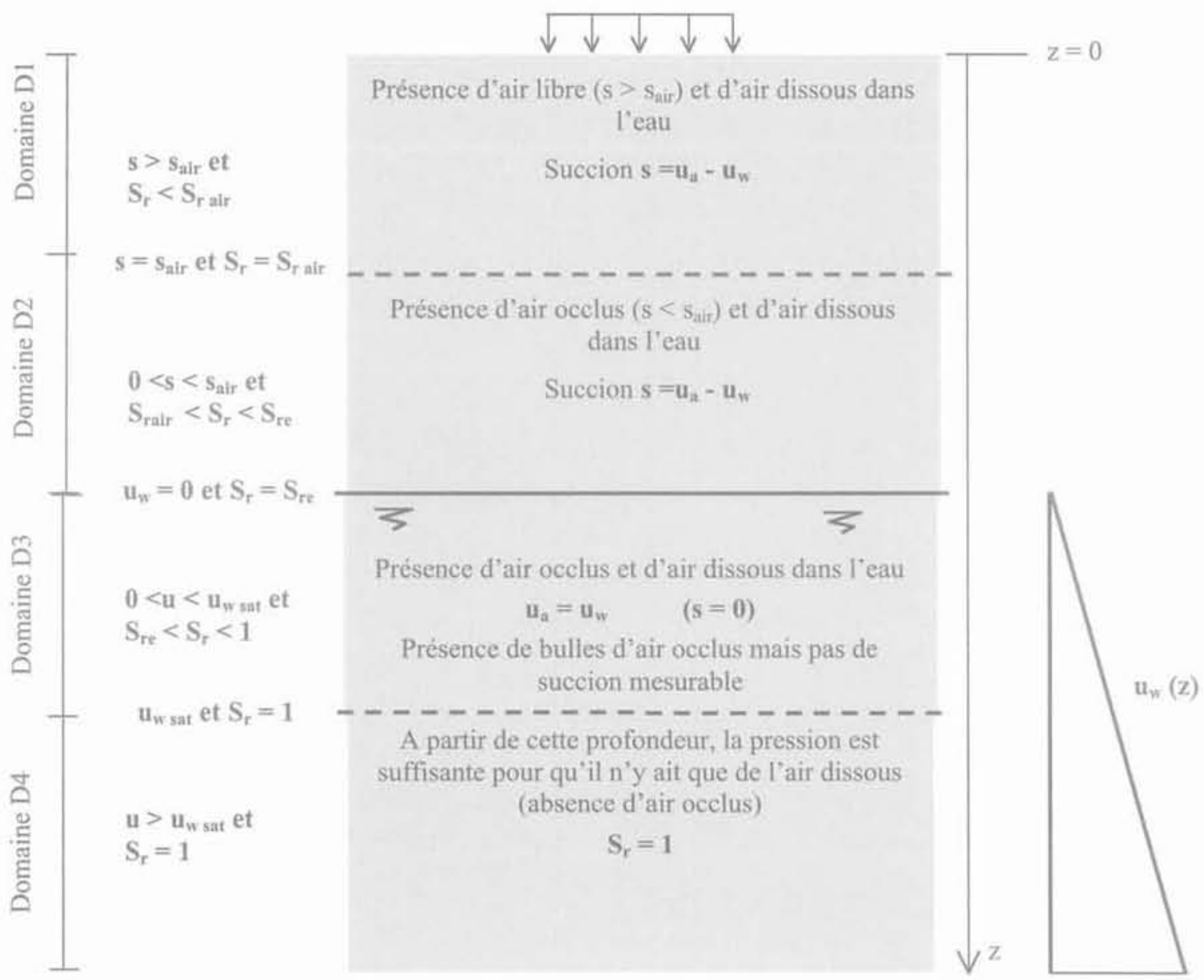

FG. 2 Représentation schématique de l'état de saturation d'un sol naturel. Schematic representation of natural soli saturation state.

cion $\left(\mathrm{s}=0\right.$ ) ce qui conduit à supposer que $\mathrm{u}_{\mathrm{u}}=\mathrm{u}_{\mathrm{u}}$, bien que des forces de tension capillaires existent à la surface de chaque bulle. De nombreux auteurs (Hilf, 1948, Li et aL, 2001) négligent ces forces et c'est Thypothèse que nous faisons par la suite dans le domaine D3.

Dans les sols compactés, le domaine D3 couvre une partie du coté humide de l'optimum Proctor (Barden, 1974).

Dans les sols naturels, le domaine D3 concerne les sols sous la nappe. On dispose de peu de mesures du degré de saturation dans ce domaine. On peut cependant citer Fourie et al. (2001), qui obtiennent des degrés de saturation compris entre $93 \%$ et $99 \%$ dans des échantillons de sables prélevés entre 6 et $16 \mathrm{~m}$ sous le toit de la nappe (technique de prélèvement par congélation); dans ces conditions, on en déduit pour ce cas particulier que $S_{r e}<93 \%$ et $99 \%$.

L'hypothèse $u_{a}=u_{\text {in }}$ semble justifiée par la taille des pores (et donc des bulles contenues dans ces pores) qui sont à l'origine de la compression volumique d'un échantillon de sol. En effet, ces pores (ou macropores entre les agrégats de particules d'argiles dans le cas des sols argileux) ont un rayon qui est bien souvent de l'ordre de $10 \mu \mathrm{m}$. Dans ces conditions, si l'on considère que les bulles emprisonnées ont la taille de ces pores ou macropores, la loi de Jurin permet de calculer une succion de l'ordre de $15 \mathrm{kPa}$ qui conduit à $\mathrm{u}_{2}=\mathrm{u}_{\mathrm{w}}+15 \mathrm{kPa}$.
Compte tenu que, dans le domaine D3, la pression d'eau absolue est $\mathrm{u}_{\mathrm{u}}+\mathrm{P}_{\mathrm{u}}>\mathrm{P}_{\mathrm{u}}=100 \mathrm{kPa}$ (pression atmosphérique), I'hvpothèse $u_{=}=u$ faite précédemment conduit à une erreur maximum de $15 \%$ sur le calcul de u dans le domaine D3 pour $u_{i}=0$. Pour $u_{w}=100 \mathrm{kPa}$, cette erreur n'est plus que de $7 \%$.

La démonstration ci-avant est justifiée par les mesures suivantes trouvées dans la littérature:

1) Pour les sols compactés selon les modes opératoires permettant de déterminer l'OPN

Pour les limons de Jossigny, Delage et Cui (2000) observent que :

- les échantillons élaborés côté sec présentent des macropores de 30 m de diamètre;

- dans les échantillons élaborés à l'optimum Proctor, seuls les grands pores de $60 \mu \mathrm{m}$ sont pleins d'air.

Dans un mélange de silt et de kaolinite $(70 \% / 30 \%$ ou $90 \% / 10 \%$ ), Garcia-Bengochea et al. (1979) observent des macropores de $3 \mu \mathrm{m}$ à $7 \mu \mathrm{m}$ de diamètre suivant la position par rapport à l'optimum Proctor; le diamètre des macropores diminue lorsque l'énergie du compactage augmente.

Sridharan et al. (1971) et Delage et Fry (2000) observent, pour une kaolinite compactée, que le rayon des pores qui gouvernent la compressibilité volumique côté sec est de l'ordre de $10 \mu \mathrm{m}$. 
Gens et Alonso (1992), citant Atabek et al. (1991), montrent que pour l'argile FoCa compactée le diamètre des macropores est de $6 \mu \mathrm{m}$, quelle que soit la teneur en eau d'élaboration de l'échantillon.

\section{2) Pour des sols remaniés}

Nagaraj et Muria (2001) mesurent des macropores de l'ordre de $20 \mu \mathrm{m}$ de diamètre à la limite de liquidité quelle que soit la limite de liquidité de l'argile. Ces auteurs estiment que le comportement mécanique des argiles est gouverné par les macropores existant entre les agrégats de particules d'argiles.

Al-Mukhtar (1995) obtient des diamètres de macropores supérieurs à $10 \mu \mathrm{m}$ sur de la kaolinite saturée et compactée statiquement à $0,3 \mathrm{MPa}$. Sous une contrainte de $1 \mathrm{MPa}$, ces macropores disparaissent.

3) Pour les sols naturels non remaniés:

Sills et al. (1991) montrent un exemple de bulle de gaz de plus de $25 \mu \mathrm{m}$ de diamètre prélevé dans les argiles silteuses de la mer d'Irlande. D'autres exemples cités dans le mème article donnent des diamètres de macropores compris entre 6 et $300 \mu \mathrm{m}$.

\section{4) Considérations physiques}

Murray (2002) et Vaughan (2003) montrent que les petites bulles ont tendance à disparaître au profit des grandes bulles par des phénomènes de diffusion locale. Les bulles d'air ont donc tendance à occuper les pores les plus importants (macropores).

Enfin, les fortes succions d'entrée d'air s, obtenues dans les sols argileux ne sont pas contradictoires avec des rayons de macropores de l'ordre de $10 \mu \mathrm{m}$. En effet, si la succion d'entrée d'air est supérieure à celle calculée par la loi de Jurin avec un rayon de $10 \mu \mathrm{m}$ (de l'ordre de $15 \mathrm{kPa}$ ), cela veut simplement dire que les macropores ne sont pas connectés entre eux.

\section{- Domaine D4 caractérisé par $\mathrm{s}=0$ et $\mathrm{S}_{\mathrm{r}}=1$ :}

Il n'y a pas d'air à l'état gazeux dans l'échantillon de sol considéré. Le sol est parfaitement saturé.

La limite entre le domaine D3 et D4 peut également $s^{\prime}$ exprimer à travers la pression interstitielle $u_{w \text { sat }}$ pour laquelle $S_{r}=1$.

La pression interstitielle $u_{w}$ correspond à la contre pression qu'il est nécessaire d’appliquer pour saturer un échantillon à l'appareil triaxial.

Dans le cas d'un sol naturel, les limites entre les différents domaines sont représentées schématiquement sur la figure 2. Dans les quatre domaines, une certaine quantité d'air est dissoute dans l'eau d'après la loi de Henry. Les limites entre les différents domaines (caractérisées par les variables $\mathrm{u}_{\mathrm{w} \text { sar }} \mathrm{S}_{\mathrm{re}} \mathrm{S}_{\mathrm{r} \text { gir }} \mathrm{S}_{\mathrm{ani}}$ ) dépendent vraisemblablement de la compacité du matériau et de son histoire géologique.

\section{2.}

\section{Les hypothèses de calcul}

Dans la suite, nous développons une théorie pour être en mesure de calculer le tassement ou le gonflement différé d'un sol (consolidation en fonction du temps). Dans le domaine D3, la compressibilité du fluide interstitiel liée à la présence de bulles d'air occlus est prise en compte.

Les hypothèses sont les suivantes:
1) L'air contenu dans le sol obéit à la loi des gaz parfaits. Pour une température T donnée et un volume d'air libre dans le sol $\mathrm{V}_{\mathrm{al}}$ soumis à une pression $\mathrm{u}_{\mathrm{i}}$, on $\mathrm{a}$ :

$$
\left(\mathrm{u}_{\mathrm{a}}+\mathrm{P}_{\mathrm{d}}\right) \cdot \mathrm{V}_{\mathrm{al}}=\text { constante }(\mathrm{T})=\mathrm{n}_{\mathrm{gar}} \cdot \mathrm{R} \cdot \mathrm{T}
$$

$\mathrm{n}_{\mathrm{onz}}$ est le nombre de moles de gaz.

2) Le volume d'air dissous dans l'eau est donné par la loi d'Henry:

$$
V_{\text {ad }}=V_{w} \cdot h
$$

avec $V_{\text {, volume qu'occuperait l'air sous la pression } u}$ s'il n'était pas dissous, $V_{w}$ volume d'eau de l'élément dé sol considéré et h constante de Henry, défini par la loi de Mariotte:

$$
\mathrm{h}=0,02 \mathrm{~T} / \mathrm{T}_{0}
$$

avec $\mathrm{T}$ température en Kelvin $\left({ }^{\circ} \mathrm{K}\right)$ et $\mathrm{T}_{0}=293^{\circ} \mathrm{K}$.

On observe que le volume d'air dissous dans l'eau $\mathrm{V}_{\mathrm{ad}}$ est indépendant de la pression $\mathrm{u}_{\mathrm{a}}$. En revanche, la loi cles gaz parfaits indique que - à volume et à température donnés - la masse d'air dissoute dépend de $u_{\text {s. }}$

Black et Lee (1973), Li et al. (2001) montrent que lorsque la pression d'air varie, la dissolution n'est pas instantanée. Suivant les cas étudiés et la rapidité de la sollicitation, la loi d'Henry sera prise en compte ou sera négligée.

3) Le rapport masse d'air (libre + dissous) sur masse d'eau est constant dans le "fluide interstitiel» (eau libre + air). Si l'on néglige la déformation du squelette, cela revient à dire qu'il n'y a pas de diffusion de l'air dans l'eau. La diffusion n'est donc pas prise en compte dans la suite à l'échelle macroscopique, même si elle peut exister localement à l'échelle microscopique (mécanisme de disparition des petites bulles au profit des plus grosses expliqué par Vaughan, 2003).

4) Le sol ne sort pas des domaines D2, D3 et D4. D'après Biarez et al. (1987), Modaressi et Abou-Bekr (1994), Khalili et Khabbaz (1998), Loret et Khalili (2002), le principe des contraintes effectives peut être appliqué:

$$
\sigma_{v}^{\prime}=\sigma_{y}-u_{w}
$$

5) Dans le domaine D2, le degré de saturation est une fonction univoque de la succion: S. (s). Cette hypothèse est généralement vraie tant que l'on ne rentre pas dans le domaine D1. Dès que l'on rentre dans le domaine D1, il y a une hystérésis au moment de la resaturation.

6) Les limites entre les différents domaines et en particulier les variables $u_{w}$ sar $S_{\text {ter }} S_{r}$ air $S_{\text {vir }}$ sont constantes. Cette hypothèse est valable tant que l'on ne rentre pas dans le domaine D1 (voir commentaire hypothèse $n^{\circ} 5$ )

7) Nous considérons une sollicitation du type œedométrique, caractérisée par $\mathrm{C}=\mathrm{C}$ ou $\mathrm{C}$. (voir définition dans le tableau $n^{\circ}$ 1) suivant que le sol se situe dans le domaine normalement ou surconsolidé. Le calcul est donc effectué en considérant que la déformation latérale est nulle. La variation d'indice des vides $\Delta \mathrm{e}$ lors d'un incrément de contrainte $\Delta \sigma^{\prime}$ est la suivante:

$$
\begin{aligned}
& \Delta e_{c}=-C_{s} \cdot \log \left(\begin{array}{l|l}
\sigma_{y}^{\prime}+\Delta \sigma^{\prime} \\
\sigma_{v}^{\prime}
\end{array}\right) \quad \text { si } \mid \begin{array}{l}
\sigma_{v}^{\prime}<\sigma_{p}^{\prime} \\
\sigma_{v}^{\prime}+\Delta \sigma^{\prime} \leq \sigma_{p}^{\prime}
\end{array} \\
& \Delta \mathrm{e}_{c}=-\mathrm{C}_{c}+\log \left(\begin{array}{l|l}
\sigma_{v}^{\prime}+\Delta \sigma^{\prime} \\
\sigma_{v}^{\prime}
\end{array}\right) \text { si } \mid \begin{array}{l}
\sigma_{p}^{\prime}<\sigma_{v}^{\prime} \\
\sigma_{p}^{\prime} \leq \sigma_{y}^{\prime}+\Delta \sigma^{\prime}
\end{array}
\end{aligned}
$$


Ce qui donne l'expression suivante en généralisant:

$$
\Delta e_{c}=-C_{x} \cdot \log \left(\frac{\sigma_{v}^{\prime}+\Delta \sigma^{\prime}}{\sigma_{v}^{\prime}}\right)
$$

La démarche est similaire sur un chemin de compression isotrope (que l'on peut obtenir au triaxial par exemple).

8) On considère que les incréments de charge $\Delta \sigma$ sont faibles par rapport à $\sigma_{v}^{\prime}$. Cette hypothèse permet en effet de linéariser la relation contrainte/déformation.

9) La compressibilité de l'eau est a et les grains solides sont supposés incompressibles par rapport à l'eau.

10) La perméabilité verticale $k$ dans les domaines D3 et D4 est reliée à I'indice des vides e par la relation suivante (Tavenas et al., 1983; Leroueil et al., 1985 , Nagaraj et Miura, 2001):

$$
e=e_{k}+c_{k} \cdot \log \left(k_{k}\right)
$$

Les constantes $e_{k}$ et $c_{k}$ peuvent ètre facilement déterminées à partir des valeurs de $c_{\text {y }}$ d'essais cedométriques standard (essai de compressibilité par paliers, cf. Magnan et al,, 1985),

11) La perméabilité verticale $k$, dans le domaine D2 est égale à celle des domaines D3 et D4. Ce point est observé expérimentalement par de nombreux auteurs (Ed Diny et al., 1993; Fredlund et Rahardjo, 1993, citant Brooks et Corey, 1964).

12) La variation d'indice des vides liée au fluage du

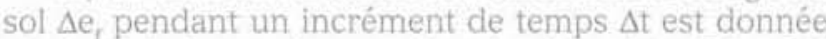
par les relations suivantes dans les domaines D3 et D4 (Magnan et al., 1979; Leroueil et al., 1985):

$$
\begin{aligned}
& \Delta \mathrm{e}_{\mathrm{c}}=-\mathrm{C}_{\mathrm{ae}} \cdot \log \left(\frac{\Delta \mathrm{t}+\mathrm{t}_{\mathrm{f}}}{\mathrm{t}_{\mathrm{f}}}\right) \\
& \mathrm{t}_{\mathrm{f}}=10 \frac{\mathrm{C}_{\mathrm{c}} \cdot \log \left(\frac{\sigma_{p}^{\prime}}{\sigma_{\mathrm{v}}^{\prime}}\right)-C_{\mathrm{s}} \cdot \log \left(\frac{\sigma_{p}^{\prime}}{\sigma_{y}^{\prime}}\right)}{C_{\alpha e}} \text { si } \mathrm{t}_{\mathrm{f}}>1 \text { jour } \\
& \mathrm{C}_{\mathrm{a}} \text { est l'indice de fluage. }
\end{aligned}
$$

13) Le comportement au fluage démontré dans la littérature pour les domaines D3 et D4 est étendu au domaine D2 où le principe des contraintes effectives est conservé.

\section{3}

\section{Les résultats théoriques}

\section{Q4as}

\section{Relations donnant le degré de saturation en fonction de la pression d'air}

Avec les hypothèses $1,2,3$ et 6 définies au $\$ 2.2$, il est possible d'établir l'équation différentielle suivante entre le degré de saturation $\mathrm{S}$ et la pression d'air $\mathrm{u}_{2}$ (Boutonnier et Virollet, 2003):

$$
\frac{d S_{r}}{S_{r}-S_{r}^{2} \cdot(1-h)}=\frac{d u_{a}}{u_{a}}
$$

Si la sollicitation est trop rapide pour que l'air puisse se dissoudre dans l'eau, cette équation peut être utilisée en prenant $h=0$.

En considérant que l'eau est saturée en air (loi de Henry), cette équation peut être intégrée. Les résultats obtenus sont les suivants:

$$
S_{p}=\frac{S_{r e}}{S_{r e} \cdot(1-h)+\frac{P_{a}}{u_{a}+P_{u}} \cdot\left[1-S_{r e} \cdot(1-h)\right]}
$$

si $\mathrm{u}_{\mathrm{a}}<0$ : domaine D2

$$
S_{t}=\frac{\frac{u_{a}+P_{a}}{P_{a}} \cdot \frac{S_{r c}}{1-S_{r e} \cdot(1-h)}}{1+\frac{u_{n}+P_{a}}{P_{a}} \cdot \frac{S_{r e} \cdot(1-h)}{1-S_{r e} \cdot(1-h)}}
$$

si $\mathrm{u}_{\mathrm{a}} \geq 0$ et $\mathrm{u}_{\mathrm{u}} \leq \mathrm{u}_{\mathrm{wath}}$ : domaine D3

$$
\text { avec } \quad u_{\text {wsat }}=\frac{P_{a} \cdot\left(1-S_{r e}\right)}{S_{r e} \cdot h}
$$

\section{Bas?}

Relation donnant le coefficient de compressibilité du fluide interstitiel équivalent

Dans le domaine D3, il est possible de définir le coefficient de compressibilité du fluide interstitiel équivalent:

$$
\mathrm{a}_{\text {int }}=\frac{\Delta \mathrm{V}_{\mathrm{y}}}{\mathrm{V}_{\mathrm{v}} \cdot \Delta \mathrm{u}_{\mathrm{w}}}
$$

Avec les mêmes hypothèses qưau \$ 3.1, on obtient (Boutonnier et Virollet, 2003):

$$
a_{\text {int }}=\frac{1-S_{r} \cdot(1-h)}{u_{w}+P_{n}} \quad\left(0<u_{w}<u_{w s a t}\right)
$$

S. étant donné en fonction de $u_{w}$ par l'équation (13) car $\mathrm{u}_{\mathrm{u}}=\mathrm{u}_{\mathrm{w}}$ dans le domaine D3.

Lors d'un chargement rapide, il est possible de calculer $\mathrm{S}$, à partir de l'équation (11) en posant h=0 et dans ces conditions l'équation (16) peut être utilisée.

Lorsque la pression interstitielle $u_{\mathrm{w}}$ devient supérieure à u..., il n'y a plus de bulles d'air dans le fluide interstitiel et le coefficient de compressibilité du fluide interstitiel est celui de l'eau:

$$
a_{i n t}=a_{w}\left(u_{w} \geq u_{w s a n}\right)
$$

Dans ces conditions, connaissant h et $\mathrm{S}$, il est possible de calculer le coefficient de compressibilité du fluide interstitiel équivalent dans les domaines D3 et D4.

La figure 3 donne le coefficient de compressibilité $\mathrm{a}_{\text {int }}$ en fonction de $\mathrm{u}_{\mathrm{w}}$ pour différentes valeurs de $\mathrm{S}_{\mathrm{rt}}$ avec

$-\mathrm{h}=0,02$ calculé pour une température de $20^{\circ} \mathrm{C}$ en utilisant l'équation (3):

- pression atmosphérique de l'air $\mathrm{P}_{\mathrm{a}}=100 \mathrm{kPa}^{-1}$;

- coefficient de compressibilité de l'eau $\mathrm{a}_{w}=5.10^{-7} \mathrm{kPa}^{-1}$. 
Afin d'analyser l'influence de la loi de Henry sur ce coefficient de compressibilité, les courbes ont également été tracées en considérant que l'air ne peut pas se dissoudre dans l'eau $(\mathrm{h}=0)$.

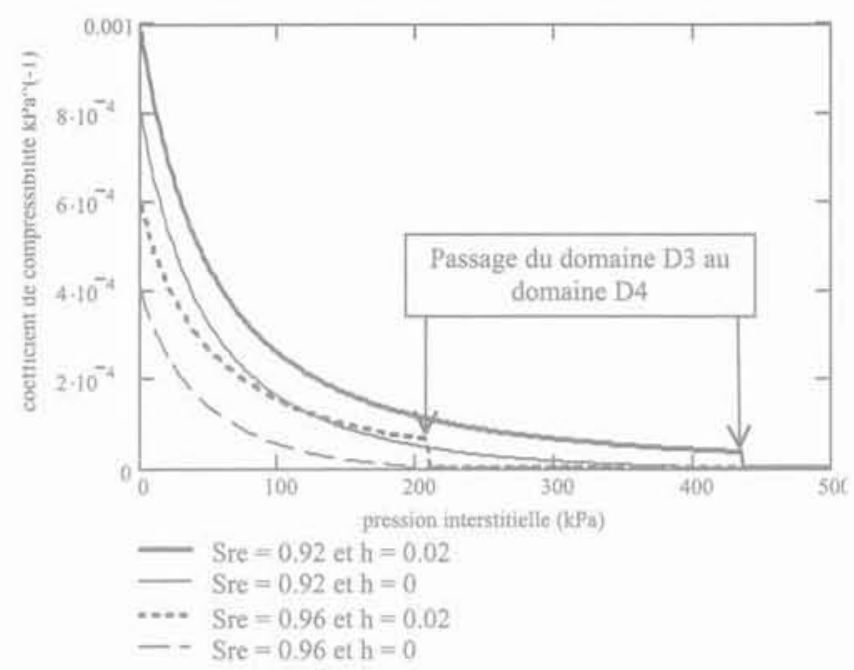

FG.3 Coefficient de compressibilité du fluide interstitiel équivalent pour $\mathrm{S}_{\mathrm{rw}}=0,92-0,96 \mathrm{et}$ $\mathrm{h}=0-0,02$ (d'après Boutonnier et Virollet. 2003).

Compressibility coefficient of equivalent pore

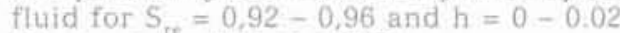
(Boutonnier and Virollet, 2003).

Les courbes de la figure 3 conduisent aux observations suivantes:

- la discontinuité du coefficient de compressibilité du fluide interstitiel observée sur les courbes correspond au passage du domaine D3 au domaine D4. La pression interstitielle correspondante est $u_{\text {was }}$ (équation (14)) dont les valeurs sont respectivement $\mathrm{u}_{\mathrm{wsat}}=435 \mathrm{et}$ $208 \mathrm{kPa}$ pour $\mathrm{S}_{r w}=0,92$ et 0,96 ;

- les valeurs de $u_{\text {wsat }}$ obtenues tendent à prouver qu'une épaisseur importante de sol sous la nappe (plusieurs dizaines de mètres) n'est pas saturée:

- les courbes avec prise en compte ou non de l'effet différé de la loi d'e Henry sont similaires pour les faibles pressions interstitielles. Aussi, la compressibilité du fluide interstitiel est principalement liée à la compressibilité des bulles de gaz (loi des gaz parfaits) dans le domaine D3.

\section{5xas}

\section{Théorie de la consolidation dans les domaines D3 et D4}

Nous démontrons ci-dessous la théorie de la consolidation prenant en compte le fluage et la compressibilité du fluide interstitiel dans les domaines D3 et D4. Des relations similaires ont déjà été établies lors de la mise au point du programme CONMULT du LCPC (Magnan et al., 1979). Les hypothèses nécessaires pour établir ces équations sont les hypothèses $n^{\text {as }} 1,2,3,4,6$, $7,8,9$ et 12 du $\$ 2.2$.

Pour un volume de sol d'épaisseur $\Delta z$ et de surface unité, le volume d'eau expulsé $\Delta \mathrm{V}_{\mathrm{iv}}$ pendant un temps $\Delta$ t est le suivant:

$$
\Delta V_{w}=(v(z+\Delta z)-v(z)) \cdot \Delta t
$$

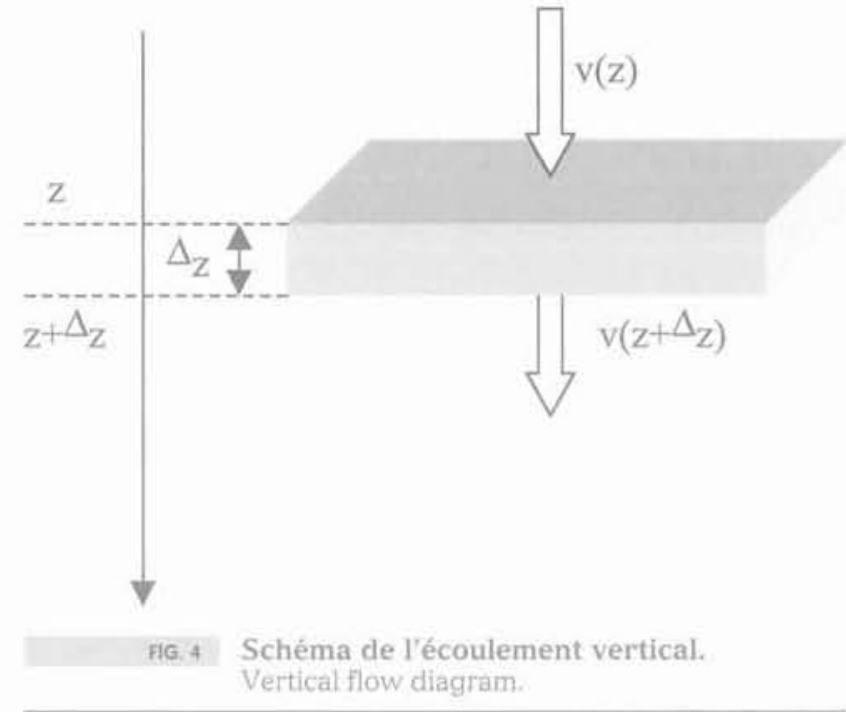

$$
v=-k_{v} \cdot \operatorname{grad}(i)=-k_{v^{*}} \frac{d\left(-z+\frac{u_{w}}{\gamma_{w}}\right)}{d z}
$$

d'où

$$
\Delta \mathrm{V}_{\mathrm{w}}=-\frac{\mathrm{k}_{\mathrm{v}}}{\gamma_{\mathrm{w}}} \cdot \frac{\delta^{2} \mathrm{u}_{\mathrm{w}}}{\delta \mathrm{z}^{2}} \cdot \Delta \mathrm{z} \cdot \Delta \mathrm{t}
$$

L'équation (21) permet de relier le volume d'eau expulsée $\Delta \mathrm{V}_{\mathrm{w}}$ à la variation du volume des vides $\Delta \mathrm{V}_{\mathrm{y}}$ et à la variation du volume du fluide interstitiel par le biais du coefficient de compressibilité $\mathrm{a}_{\text {int }}$ :

$$
\Delta V_{w}=-\Delta V_{w}-a_{\text {int }} \cdot \frac{e}{1+e} \cdot \Delta z \cdot \Delta u_{w}
$$

Avec:

$$
\begin{aligned}
& \Delta \mathrm{V}_{w}=\left(\Delta \mathrm{e}_{f}+\Delta \mathrm{e}_{\mathrm{c}}\right) \cdot \frac{\Delta \mathrm{z}}{(1+\mathrm{e})} \\
& \Delta \mathrm{V}_{\mathrm{w}}=\left(-\mathrm{C}_{\alpha e} \cdot \log \left(\frac{\Delta \mathrm{t}+\mathrm{t}_{f}}{\mathrm{t}_{f}}\right)-\mathrm{C}_{\mathrm{x}} \cdot \log \left(\frac{\left.\sigma_{\mathrm{v}}^{\prime}-\Delta \mathrm{u}_{\mathrm{w}}\right)}{\sigma_{v}^{\prime}}\right)\right) \cdot \frac{\Delta \mathrm{z}}{1+\mathrm{e}} \\
& \Delta \mathrm{V}_{\mathrm{w}}=\left(-\mathrm{C}_{\alpha e} \cdot \frac{\Delta \mathrm{t}}{\ln (10) \mathrm{t}_{\mathrm{f}}}+\mathrm{C}_{\mathrm{x}} \cdot \frac{\Delta \mathrm{u}_{\mathrm{w}}}{\ln (10) \sigma_{\mathrm{v}}^{\prime}}\right) \cdot \frac{\Delta \mathrm{z}}{1+\mathrm{e}}
\end{aligned}
$$

Nota: dans l'équation (22), on fait l'hypothèse que $\Delta t<<t$. Par ailleurs, on considère que, pendant l'incrément de temps $\Delta t$, la contrainte totale est constante ce qui permet d'écrire $\Delta \sigma_{\mathrm{y}}^{\prime}=--\Delta \mathrm{u}_{\mathrm{w}}$.

En combinant les équations (20), (21) et (22), on obtient:

$$
\begin{aligned}
& \frac{\Delta \mathrm{u}_{\mathrm{w}}}{\Delta t} \cdot\left(1+\mathrm{a}_{\mathrm{int}} \cdot \frac{\mathrm{e} \cdot \sigma_{\mathrm{v}}^{\prime} \cdot \ln (10)}{\mathrm{C}_{\mathrm{x}}}\right)=\frac{\mathrm{k}_{\mathrm{v}} \cdot(1+\mathrm{e}) \cdot \sigma_{\mathrm{v}}^{\prime} \cdot \ln (10)}{\gamma_{\mathrm{w}} \cdot \mathrm{C}_{\mathrm{x}}} \cdot \frac{\delta^{2} \mathrm{u}_{\mathrm{w}}}{\delta \mathrm{z}^{2}} \\
& +\frac{\mathrm{C}_{\alpha \mathrm{e}} \cdot \sigma_{y}^{\prime}}{\mathrm{C}_{\mathrm{x}}} \cdot \frac{1}{\mathrm{t}_{\mathrm{f}}}
\end{aligned}
$$


Lorsque $\Delta t \rightarrow 0$, on obtient

$\frac{\delta u_{w}}{\delta_{i}} \cdot\left(1+a_{i n t} \cdot \frac{e \cdot \sigma_{y}^{\prime} \cdot \ln (10)}{C_{x}}\right)=\frac{k_{v} \cdot(1+e) \cdot \sigma_{y}^{\prime} \cdot \ln (10)}{\gamma_{w} \cdot C_{x}} \cdot \frac{\delta^{2} u_{w}}{\delta z^{2}}$ $+\frac{C_{\alpha e} \cdot \sigma_{v}^{\prime}}{C_{x}} \cdot \frac{1}{4}$

Si le terme de fluage et la compressibilité du fluide interstitiel sont nuls $\left(C_{a t}=0\right.$ et $\left.a_{i n t}=0\right)$, on retrouve l'équation de la consolidation classique avec le coefficient de consolidation verticale c donné par l'équation (25) pour les domaines D3 et D4:

$$
c_{v_{-} D 3 D 4}=\frac{k_{v} \cdot(1+e) \cdot \sigma_{v}^{\prime} \cdot \ln (10)}{\gamma_{w} \cdot C_{x}}
$$

\section{4}

\section{Théorie de la consolidation dans le domaine D2}

Nous démontrons ci-dessous la théorie de la consolidation verticale prenant en compte le fluage et la compressibilité du fluide interstitiel dans le domaine D2. Les hypothèses nécessaires pour établir ces équations sont les hypothèses $n^{\text {as }} 1,2,3,4,5,6,7,8,9,11,12$ et 13 du $\$ 2.2$.

Les conventions de signes sont données par la figure 4.

La variation du degré de saturation $\Delta S$, pendant l'intervalle de temps $\Delta t$ est fonction:

- de la quantité d'eau expulsée $\Delta \mathrm{V}_{\mathrm{w}}$ de la tranche de sol d'épaisseur $\Delta z$;

- de la variation du volume des vides $\Delta \mathrm{V}$, de la tranche de sol.

$$
\Delta S_{r}=-\frac{1+e}{e \cdot \Delta z} \cdot\left(\Delta V_{w}+S_{r} \cdot \Delta V_{v}\right)
$$

Cornpte tenu des hypothèses prises en compte, les équations (20) et (22) restent valables et, combinées à l'équation (26), elles permettent d'obtenir:

$$
\begin{aligned}
& \frac{\Delta S_{s}}{\Delta t}+\frac{S_{r} \cdot C_{x}}{e \cdot \sigma_{v}^{\prime} \cdot \ln (10)} \cdot \frac{\Delta u_{w}}{\Delta t}= \\
& \frac{k_{v} \cdot(1+e)}{\gamma_{w} \cdot e} \cdot \frac{\delta^{2} u_{w}}{\delta z^{2}}+\frac{S_{r} \cdot C_{\alpha c}}{e \cdot t_{f} \cdot \ln (10)}
\end{aligned}
$$

Lorsque $\Delta t \rightarrow 0$, on obtient:

$$
\begin{aligned}
& \frac{\delta S_{r}}{\delta t}+\frac{S_{r} \cdot C_{x}}{e \cdot \sigma_{v}^{\prime} \cdot \ln (10)} \cdot \frac{\delta u_{w}}{\delta t}= \\
& \frac{k_{v} \cdot(1+e)}{\gamma_{w} \cdot e} \cdot \frac{\delta^{2} u_{w}}{\delta z^{2}}+\frac{S_{r} \cdot C_{\alpha v}}{e \cdot t_{f} \cdot \ln (10)}
\end{aligned}
$$

Par ailleurs, I'hypothèse $n^{\circ} 5$ du $\$ 2.2$ nous permet d'écrire:

$$
\begin{array}{r}
\frac{d S_{r}(s)}{d t}=\frac{d S_{r}}{d s} \cdot \frac{d s}{d u_{w}} \cdot \frac{d u_{w}}{d t}= \\
\frac{d S_{r}}{d s} \cdot\left(\frac{d u_{a}}{d u_{w}}-1\right) \cdot \frac{d u_{w}}{d t}
\end{array}
$$

En combinant les équations (28) et (29), on obtient:

$$
\frac{\delta u_{w}}{\delta t}=\frac{\frac{k_{v} \cdot(1+e)}{\gamma_{w} \cdot e}}{\frac{d S_{r}}{d s} \cdot\left(\frac{d u_{u}}{d u_{w}}-1\right)+\frac{S_{r} \cdot C_{x}}{e \cdot \sigma_{v}^{\prime} \cdot \ln (10)}} \cdot \frac{\delta^{2} u_{w}}{\delta z^{2}}+
$$

avec:

dS $\quad$; fonction de la succion s connue car $S_{r}$ est une ds fonction des:

$\mathrm{du}_{\mathrm{a}}$ : donné par l'équation (31) déjà établie par Bou$\frac{d u_{w}}{d u_{w}}$ tonnier et Virollet (2003);

$$
\frac{d u_{\mathrm{a}}}{d u_{\mathrm{w}}}=\frac{\frac{-d S_{r}}{d s} \cdot\left(u_{\mathrm{a}}+P_{\mathrm{n}}\right)}{\frac{-d S_{r}}{d s} \cdot\left(u_{\mathrm{a}}+P_{\mathrm{a}}\right)+S_{r} \cdot\left[1-S_{r} \cdot(1-h)\right]}
$$

Si le terme de fluage est nul dans l'équation (30) $\left(C_{\text {cy }}\right.$ $=0$ ), on trouve une forme d'équation correspondant a l'équation de la consolidation avec le coefficient de consolidation verticale $c_{v}$ donné par l'équation (32) pour le domaine n?

$$
c_{v_{-} D 2}=\frac{\frac{k_{v} \cdot(1+e)}{\gamma_{w} \cdot e}}{\frac{d S_{r}}{d s} \cdot\left(\frac{d u_{\mathrm{a}}}{d u_{w}}-1\right)+\frac{S_{r} \cdot C_{X}}{e \cdot \sigma_{v}^{i} \cdot \ln (10)}}
$$

Il est intéressant de noter que les équations (33) et (34) permettent de relier le coefficient de consolidation verticale dans le domaine D2 ( $\propto$ côté succion $\cdots \mathrm{c}_{\mathrm{v}} \mathrm{p}$ ) au coefficient de consolidation verticale dans les domaines D3 et D4 ( $\alpha$ sous la nappe $x, c_{v-D 3 p 4}$ cf. définition équation (25)).

avec:

$$
c_{v_{-D 2}}=\Gamma \cdot c_{v, D 3 D 4}
$$

$$
\Gamma=\frac{1}{\frac{d S_{f}}{d s} \cdot\left(\frac{d u_{a}}{d u_{w}}-1\right) \cdot \frac{e \cdot \sigma_{v}^{\prime} \cdot \ln (10)}{C_{x}}+S_{r}}
$$


Compte tenu des équations (12) et (31), le coefficient $\Gamma$ est fonction de $\frac{d S_{F}}{d s}, u_{x^{\prime}} S_{r x^{\prime}}$ h et $\frac{e . \sigma_{\gamma}^{\prime} \cdot \ln (10)}{C_{x}}$

Par ailleurs, on vérifie que $\mathrm{T}>0$ car $\frac{\mathrm{d} S_{r}}{\mathrm{ds}}<0$ lle degré de saturation diminue lorsque la succion augmente) et $\frac{\mathrm{du}}{\mathrm{du}_{\mathrm{w}}}-1<0$ (cf. équation (31)).

Afin d'estimer l'intervalle de variation du coefficient $\Gamma$, nous avons cherché clans un premier temps à évaluer la variation de $\Gamma$ en fonction de $u_{a}$, les autres paramètres $\frac{\mathrm{dS}}{\mathrm{ds}}, \mathrm{S}_{r e^{\prime}}$, h et $\frac{\mathrm{e} \cdot \sigma_{\mathrm{v}}^{\prime} \cdot \ln (10)}{\mathrm{C}_{\mathrm{x}}}$ restant constants. La pression d'air $u_{u}$ est comprise entre 0 (correspondant à la limite entre les domaines D2 et D3, succion $s=0$, $\mathrm{u}_{\mathrm{a}}=\mathrm{u}_{\mathrm{v}}=0, \mathrm{~S}_{\mathrm{r}}=\mathrm{S}_{\mathrm{rx}}$ ) et $\mathrm{u}_{\mathrm{u}}<0$ (correspondant à la limite entre les domaines D1 et D2, succion $S=S_{\text {air }}$ et $S_{r}=S_{r a r r}$ ).

Il est possible de calculer $\mathrm{u}_{\mathrm{a} \text { mir }}$ à partir de la relation univoque supposée $S$ ( $(\mathrm{s})$ (hypothèse $n^{\circ} 5$ et équation (35) ci-dessous) et de la relation $S_{r}\left(u_{\mathrm{f}}\right.$ ) (cf. équation (12)), Le calcul de la fonction $u_{a}(\mathrm{~s})$ qui permet d'obtenir $\mathrm{u}_{\text {aar }}=$ $\mathrm{u}_{\mathrm{s}}\left(\mathrm{s}_{\mathrm{air}}\right)$ fait appel à des approximations d'intégrales et des résolutions implicites d'une équation à une inconnue.

Pour les applications numériques et l'étude paramétrique présentée dans la suite, nous faisons l'hypothèse supplémentaire que $\frac{\mathrm{dS}}{\mathrm{ds}}$ est constant dans le domaine D2.

$$
S_{r}\left(S_{)}\right)=S_{r e}+\frac{S_{r}}{S_{\text {air }}} \cdot\left(S_{\text {tair }}-S_{r e t}\right)
$$

Notons que cette hypothèse de linéarité dans le domaine D2 a déjà été faite par LeBihan et Leroueil (2002) pour l'analyse des écoulements d'eau et d'air dans les barrages.

Nous présentons sur la figure $n^{\circ} 5$ un exemple de résultat obtenu:

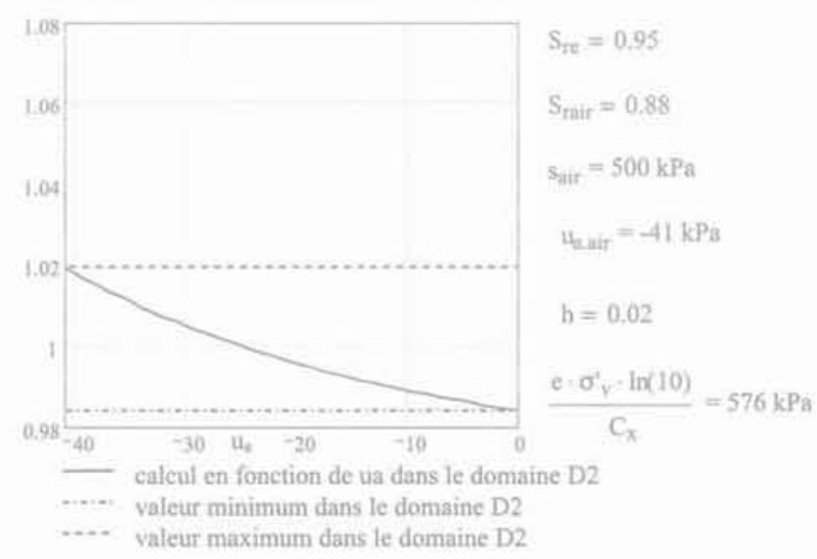

FiG.5 Exemple de résultat du calcul de $\mathrm{r}$. Result example of $\mathrm{I}$ calculation.

Étant donné les faibles variations du coefficient I dans le domaine D2, nous présentons uniquement dans la suite les valeurs minimale $\left(\Gamma_{\text {minimun }}\right)$ et maximale $\left(\Gamma_{\text {miocimur }}\right)$ prises par $\Gamma$ dans le domaine D2.
Nous présentons dans les figures 6, 7 et 8 une étude paramétrique permettant de balayer la plupart des cas courants.

La principale conclusion de cette étude paramétrique (Figs, 6, 7 et 8) est que le coefficient $\Gamma$ est généralement proche de 1, en particulier pour les faibles contraintes ou pour les sols très argileux (succion d'entrée d'air élevée). Pour les contraintes plus fortes, le coefficient $\mathrm{F}$ doit être calculé (les abaques ci-dessus peuvent être utilisées mais elles ne couvrent pas tous les cas).

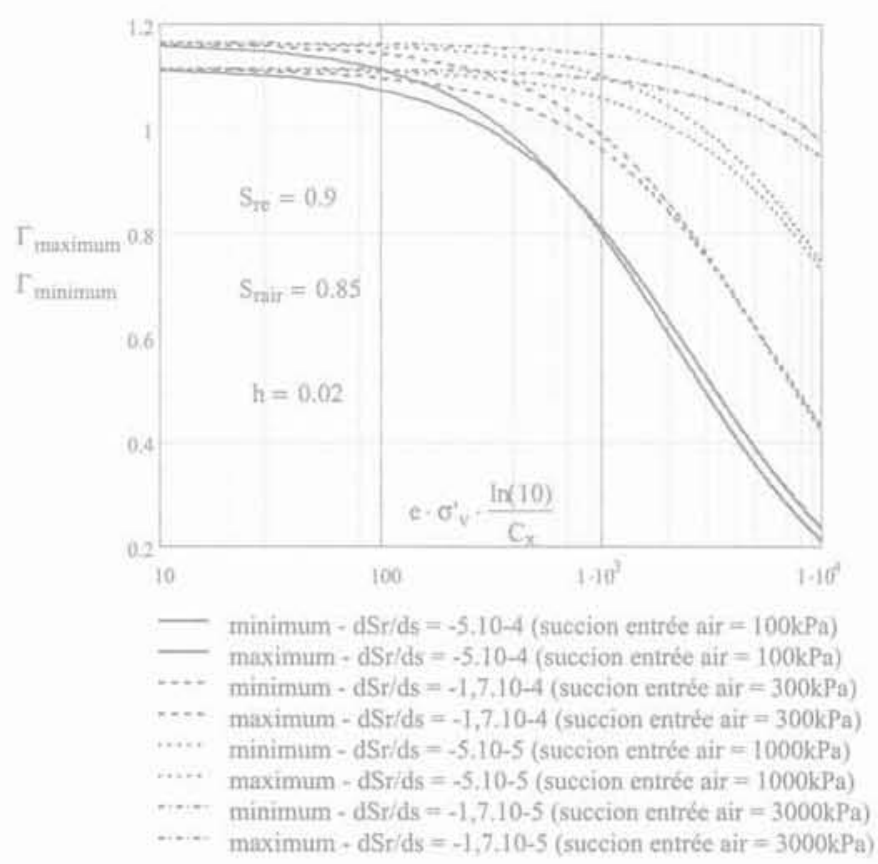

FIG.6 Abaques pour $\mathrm{S}_{r \mathrm{f}}=0,9$ et $\mathrm{S}_{\text {rair }}=0,85$. Charts for $S_{n x}=0.9$ et $S_{r=1}=0.85$.

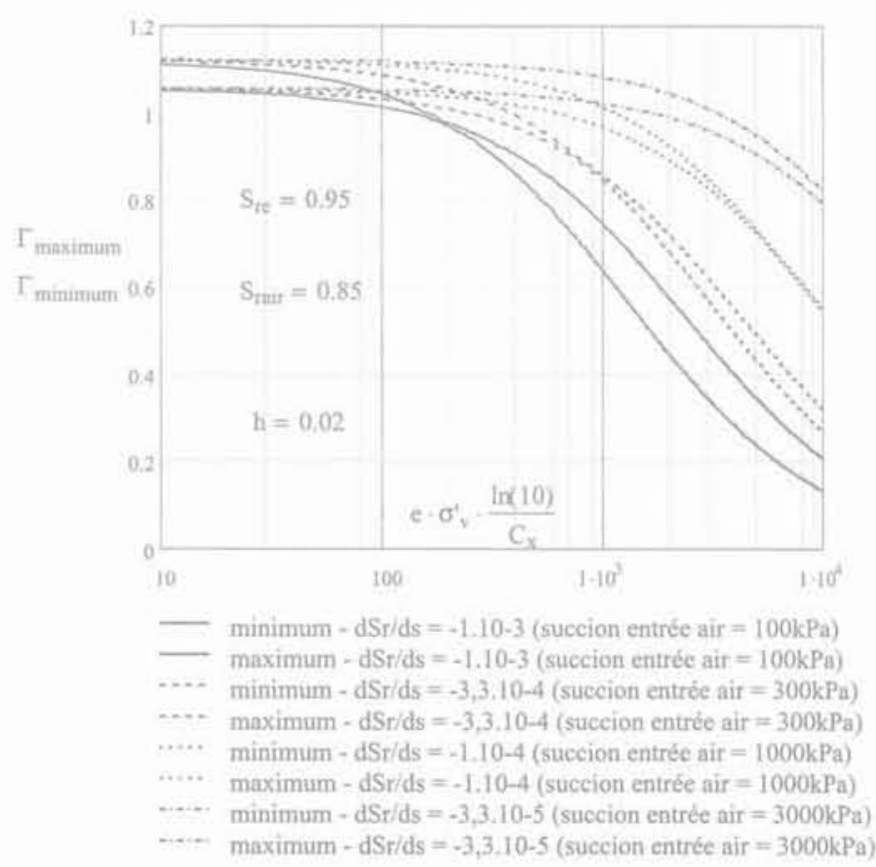

FG: 7 Abaques pour $\mathrm{S}_{\mathrm{rg}}=0,95$ et $\mathrm{S}_{\text {rar }}=0,85$. Charts for $S_{e r}=0.95$ et $S_{r \text { rut }}=0.85$.

Dans une première approche, la cinétique des phénomènes de gonflement des sols proches de la satura- 


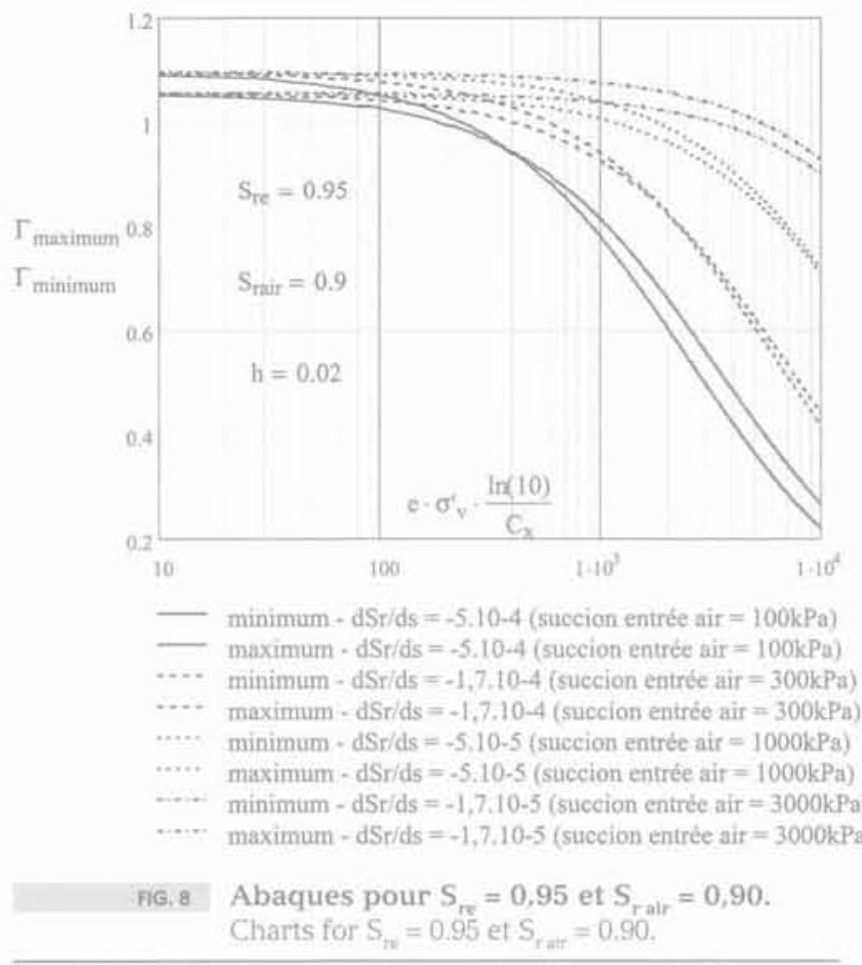

\section{Etat initia}

Au point $A$, le sol est saturé ou proche de la saturation. tion peut donc être étudiée à partir de valeurs de c déterminées dans les domaines D3/D4 ( $c_{\text {y pap4 }}$ ), c'est-àdire à l'aide d'essais œdométriques standards (essais œdométriques par paliers). Cependant, une interprétation fine est nécessaire (cf. équation (25) et la méthode d'interprétation de l'essai selon Magnan et al. (1985) pour déterminer la relation entre indice des vides et perméabilité) pour être capable de calculer la variation de $c_{\mathrm{y}}$ en fonction de la contrainte $\sigma_{\mathrm{y}}^{\prime}$.

\section{3}

\section{Exemple d'application}

\section{1}

\section{Introduction}

Boutonnier et Serratrice (2002) ont présenté les résultats d'essais spéciaux de mécanique des sols pour préciser les conditions de gonflement d'une plateforme en fond de déblai dans des marnes (Argiles argiles de la Woëvre sur la commune de Vigneulles-lès-
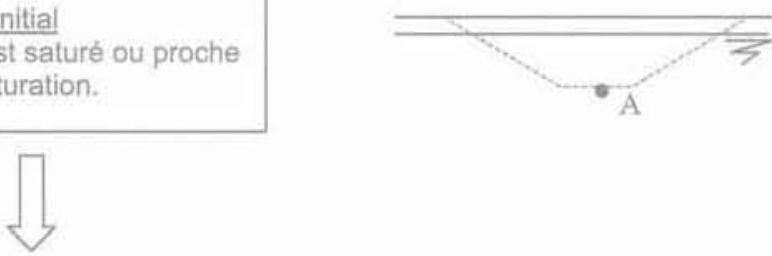

Réalisation du déblaí

Au point $A$, la pression interstitielle diminue jusqu'à devenir éventuellement négative; la succion apparaît lorsque suffisamment d'air dissous dans le fluide interstitiel repasse à l'état gazeux.

La zone intéressée par cette diminution des pressions interstitielles est importante (problème de diffusion des contraintes).

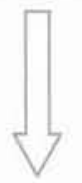

Gonflement différé après réalisation du déblai

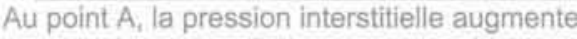
progressivement grâce aux apports d'eau superficiels (cas oủ le sol est en succion) et / ou aux écoulements dans le sol.

Ceci conduit à une diminution de la contrainte effective verticale et à un gonflement différé.

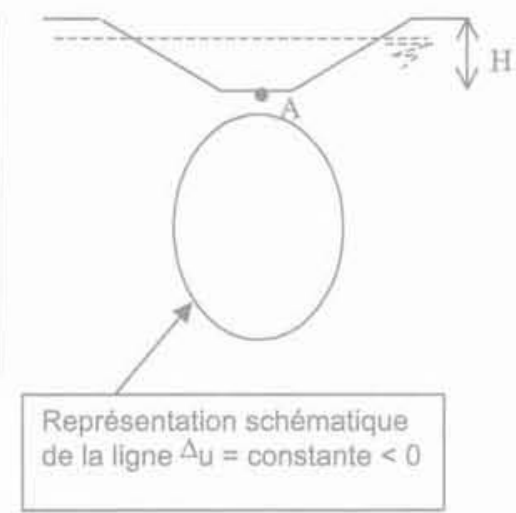

Représentation schématique de la ligne $\Delta_{U}=$ constante $<0$

$\Delta_{\mathrm{u}}$ se dissipe progressivement pour tendre vers 0

FGG.9 Représentation schématique des mécanismes liés au gonflement différé (Boutonnier et Serratrice, 2002).

Schematic representation of deferred swelling mechanisms in a cut (Boutonnier and Serratrice, 2002). 
TABLEAUII Caractéristiques moyennes d'état (d'après Boutonnier et Serratrice, 2002).

Mean state parameters (Boutonnier and Serratrice, 2002).

\begin{tabular}{|c|c|c|c|c|c|c|}
\hline Matériau & $\begin{array}{c}w \\
(\%)\end{array}$ & $\left(\mathrm{kN} / \mathrm{m}^{3}\right)$ & $(\mathrm{kN} / \mathrm{m})$ & $(\mathrm{kN} / \mathrm{m})$ & $\begin{array}{l}\text { e } \\
0\end{array}$ & $\begin{array}{c}5 \\
(\%)\end{array}$ \\
\hline $\begin{array}{l}\text { Marne } \\
\text { Marne indurée } \\
\text { Marne calcareuse }\end{array}$ & $\begin{array}{l}15,8 \\
15,7 \\
14,2\end{array}$ & $\begin{array}{l}21,6 \\
21,3 \\
21,9\end{array}$ & $\begin{array}{l}18,6 \\
18,4 \\
19,2\end{array}$ & $\begin{array}{l}26,29 \\
26,39 \\
26,50\end{array}$ & $\begin{array}{l}0,412 \\
0,434 \\
0,381\end{array}$ & $\begin{array}{r}102 \\
97 \\
100\end{array}$ \\
\hline
\end{tabular}

Hattonchâtel, département de la Meuse, France). Le projet concerné est la LGV Est (ligne ferroviaire à grande vitesse Paris-Strasbourg). Les critères de déformation sur les projets ferroviaires à grande vitesse sont en effet très stricts (gonflement nul après la mise en servicel

Un des objectifs de ces essais était d'estimer la vitesse de dissipation des gonflements en fonction du temps, comme illustré sur la figure 9 .

\section{0}

\section{Propriétés physiques de la marne}

L'ouverture des échantillons au laboratoire fait apparaître une marne compacte de couleur grise, fracturée, parfois microfissurée. Localement, il apparait des passages plus tendres et plus humides. Les matériaux prélevés, marnes, marnes indurées, marnes calcareuses, sont saturés ou très proches de la saturation.

Cinq analyses granulométriques par diffraction laser ont été réalisées et montrent une bonne homogénéité des marnes. La fraction argileuse $(\%<2 \mu \mathrm{m})$ est comprise entre 10 et $13 \%$ et $\mathrm{D}_{\text {mix }}=0,1 \mathrm{~mm}$ (diamètre des plus gros éléments). Les histogrammes des dimensions des grains marquent la présence des silts (D) $\approx 0,02 \mathrm{~mm}$ ).

\section{3}

\section{$c_{y}$ obtenus lors de la consolidation des éprouvettes sur les triaxiaux haute pression}

\section{Fis.1}

\section{Dispositif expérimental et mode opératoire}

Après découpage manuel, les éprouvettes sont montées dans les cellules triaxiales sur des plaques poreuses saturées en eau désaérée (diamètre des éprouvettes $5 \mathrm{~cm}$, élancement égal à 2). Une première phase de saturation est appliquée pendant une douzaine d'heures (confinement $320 \mathrm{kPa}$ ou $550 \mathrm{kPa}$, contre-pression $300 \mathrm{kPa}$ ou $500 \mathrm{kPa}$ ). Puis les phases de consolidation sont effectuées sous des pressions effectives isotropes p comprises entre 700 et $6450 \mathrm{kPa}$ (drainage de tête et de pied, pas de drainage latéral).

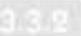

\section{Résultats obtenus}

Les résultats des consolidations sont présentés au travers des courbes $\left(\log (t), \varepsilon_{v}\right)$ où $\varepsilon_{v}$ est la déformation de volume $\left(\varepsilon_{v}=\Delta V / V_{o^{*}} V_{0}\right.$ volume initial de l'éprou-

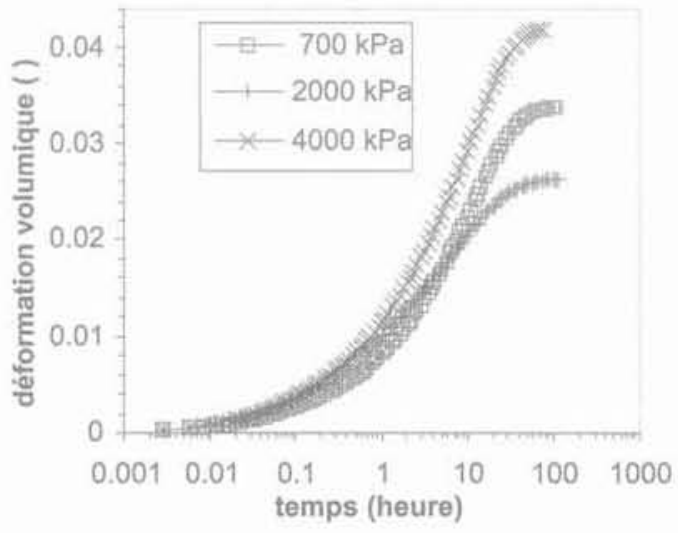

a. déformation volumique, temps

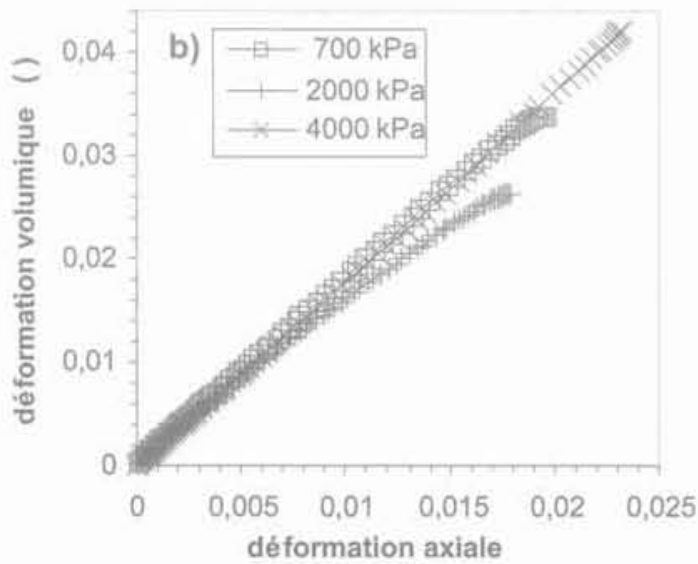

b. chemin des déformations

FIG. 10 Exemple de courbes de consolidation isotrope pour différentes contraintes de consolidation $\sigma_{3}$ (Boutonnier et Serratrice, 2002).

Example of isotropic consolidation curves for different isotropic consolidation stress $\sigma_{3}$ (Boutonnier and Serratrice, 2002). 
TABLEAUIII Consolidations isotropes. Essais triaxiaux CD (d'après Boutonnier et Serratrice, 2002). Isotropic consolidation. CD triaxial test (Boutonnier and Serratrice, 2002).

\begin{tabular}{|c|c|c|c|c|c|c|c|}
\hline Éprouvette & Type d'essai & $\begin{array}{c}\mathrm{h}_{0} \\
(\mathrm{~cm})\end{array}$ & $\stackrel{\mathrm{P}}{\text { (kPa) }}$ & $\begin{array}{l}t_{n o} \\
\text { (h) }\end{array}$ & $\frac{\varepsilon_{y c}}{0}$ & $\frac{\varepsilon_{n}}{0}$ & $\frac{\mathrm{c}_{\mathrm{y}}}{\left(\mathrm{m}^{2} / \mathrm{s}\right)}$ \\
\hline $\begin{array}{l}\text { TVS01 } \\
\text { TVS03 } \\
\text { TVS08 } \\
\text { TVS10 } \\
\text { TVS13 } \\
\text { TVS14 }\end{array}$ & compression & $\begin{array}{l}9,65 \\
9,32 \\
9,87 \\
9,86 \\
9,87 \\
9,44\end{array}$ & $\begin{array}{c}700 \\
2000 \\
700 \\
4000 \\
700 \\
2400 \\
6450\end{array}$ & $\begin{array}{l}43,3 \\
55,1 \\
11,2 \\
72,1 \\
16,1 \\
55,1 \\
37,3\end{array}$ & & $\begin{array}{l}0,0215 \\
0,0253 \\
0,0377 \\
0,0404 \\
0,0043 \\
0,0275 \\
0,0425\end{array}$ & $\begin{array}{l}1,210^{-1} \\
8,610^{-5} \\
4,710^{-6} \\
7,310^{-1} \\
3,310^{-1} \\
8,810^{-1} \\
1,310^{-6}\end{array}$ \\
\hline $\begin{array}{l}\text { TVSO2 } \\
\text { TVSO9 } \\
\text { TVS11 }\end{array}$ & extension & $\begin{array}{l}9,02 \\
8,50 \\
8,50\end{array}$ & $\begin{array}{c}2000 \\
4000 \\
700\end{array}$ & $\begin{array}{l}33,7 \\
39,9 \\
39,9\end{array}$ & $\begin{array}{l}0,0178 \\
0,0232 \\
0,0196\end{array}$ & $\begin{array}{l}0,0262 \\
0,0420 \\
0,0338\end{array}$ & $\begin{array}{l}1,310^{-1} \\
9,910^{-1} \\
9,910^{-1}\end{array}$ \\
\hline
\end{tabular}

$\sigma_{\text {i }}$ contrainte axiale maximale $1^{\prime r}$ cycle

$\sigma_{\text {man }}$ contrainte axiale d'imbibition

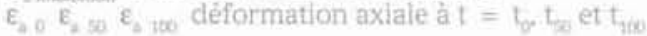

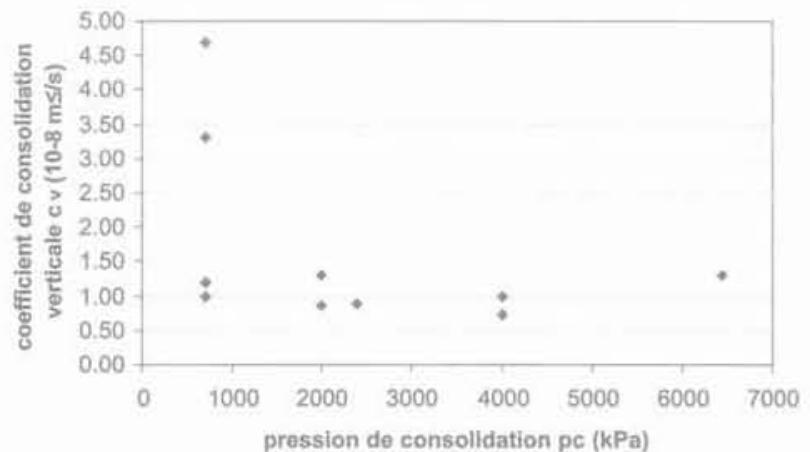

FIG,11 Valeurs de $c_{y}$ en fonction de la pression de consolidation $\mathrm{p}_{\text {. }}$.

$\mathrm{c}_{\mathrm{\gamma}}$ values versus consolidation pressure $\mathrm{p}_{\mathrm{c}}$ vette). La figure 10 montre un exemple de courbes. Pour les essais dont le cisaillement (après consolidation) est effectué en extension, la déformation axiale $\varepsilon_{\text {. }}$ est mesurée.

Les valeurs de $c_{y}$ obtenues lors des phases de consolidation isotrope (avec drainacie en tête et en pied de l'éprouvette) sont données dans le tableau III et sur la figure 11. Les valeurs de $c_{y}$ obtenues sont indépendantes de la pression de consolidation $p_{\text {c }}$ dans la gamme de contraintes testées. La valeur de $c_{y}$ plus élevẻe sur l'éprouvette TVS08 est liée à la fissuration de l'éprouvette. Si l'on exclue l'éprouvette TVS08, les valeurs de $c_{y}$ obtenues sont homogènes et la moyenne est égale à $\mathrm{c}_{\mathrm{v} \text { maven }}=1,310^{-8} \mathrm{~m}^{2} / \mathrm{s}$.

Compte tenu du mode opératoire décrit au $\$ 3.1$, les valeurs de $c_{\text {. }}$ mesurées correspondent aux domaines D3 et D4.

De manière analogue au chemin de contrainte cedométrique (cf. \$ 2.3.3), on peut établir l'expression de c pour une compression isotrope avec drainage sur les deux faces (équation 36).

$$
c_{v_{-} \text {D3D4_triax }}=\frac{k_{v} \cdot K}{\gamma_{w}}
$$

$\mathrm{K}$ est le module de compression volumique.

Les marnes testées étant très raides, il y a peu d'évolution d'indice des vides et il est donc probable que la perméabilité verticale $k_{v}$, est à peu près constante. Par ailleurs, les valeurs de $c_{v}$ obtenues au triaxial étant peu influencées par la pression de consolidation $p$, on en déduit que le matériau a un comportement quasi élastique ( $\mathrm{K}$ constant).

\section{4}

\section{c obtenus après imbibition d'éprouvettes à l'œdomètre}

\section{EMan}

\section{Dispositif expérimental et mode opératoire}

Ces essais œdométriques ont pour but de déterminer l'effet de l'imbibition et mesurer les propriétés de déchargement de la marne. Quatre éprouvettes sont

TABLEAU IV Essais cedométriques en parallèle. Mesure de c (d'après Boutonnier et Serratrice, 2002). Oedometer tests. c measurement (from Boutonnier and Serratrice, 2002).

\begin{tabular}{|c|c|c|c|c|c|c|c|c|}
\hline Eprouvette & $\begin{array}{l}0_{\text {amas }} \\
(\mathrm{kPa})\end{array}$ & $\begin{array}{l}9_{\text {a }} \text { (XPa) } \\
\text { (XPana }\end{array}$ & $\begin{array}{l}\varepsilon_{\mathrm{a}} \\
0\end{array}$ & $\begin{array}{l}\varepsilon_{350} \\
0\end{array}$ & $\begin{array}{l}t_{\text {sai }} \\
\text { (h) }\end{array}$ & $\begin{array}{c}\varepsilon_{\text {s }} \\
0\end{array}$ & $\begin{array}{l}t_{\text {hon }} \\
\text { (b) }\end{array}$ & $\begin{array}{c}\mathrm{c}_{\mathrm{y}} \\
\left(\mathrm{m}^{2} / \mathrm{s}\right)\end{array}$ \\
\hline TVSO4 & 201 & 48 & 0,01472 & 0,01006 & 0,635 & 0,00539 & 4,0 & $1,310^{-8}$ \\
\hline TVSO5 & 205 & 101 & 0,01836 & 0,01421 & 0,432 & 0,01005 & 3,4 & $1,910^{-8}$ \\
\hline
\end{tabular}




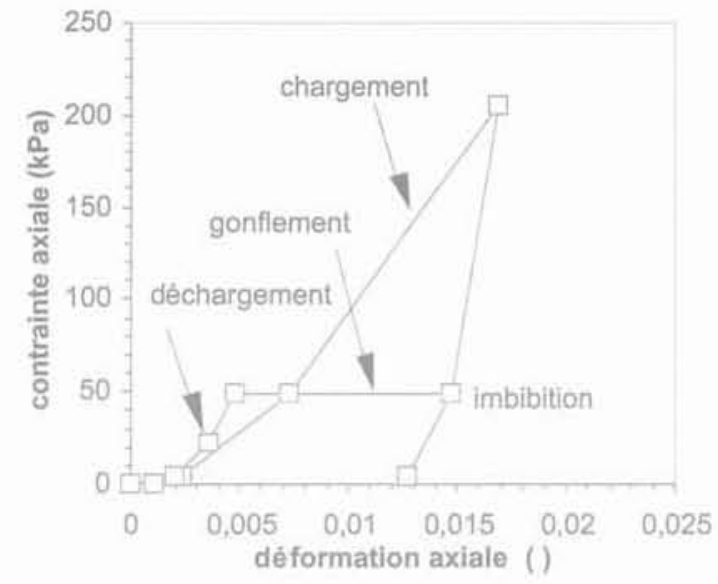

a. imbibition à $48 \mathrm{kPa}$

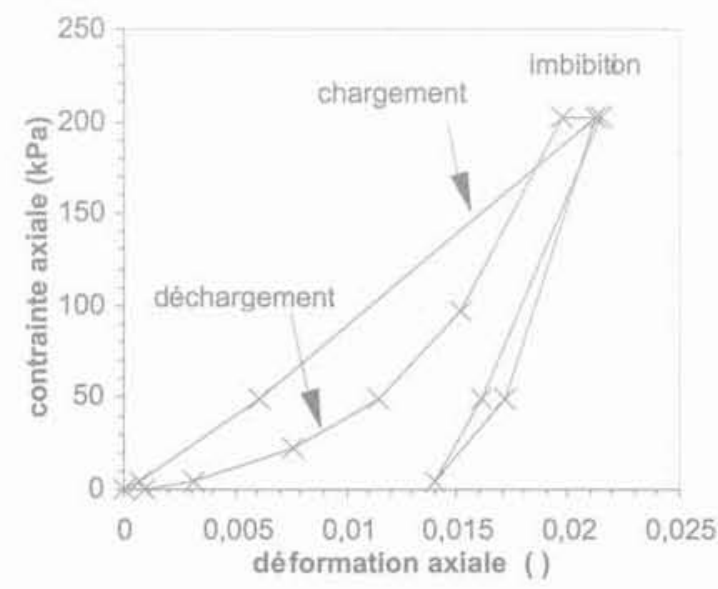

c. imbibition à $202 \mathrm{kPa}$

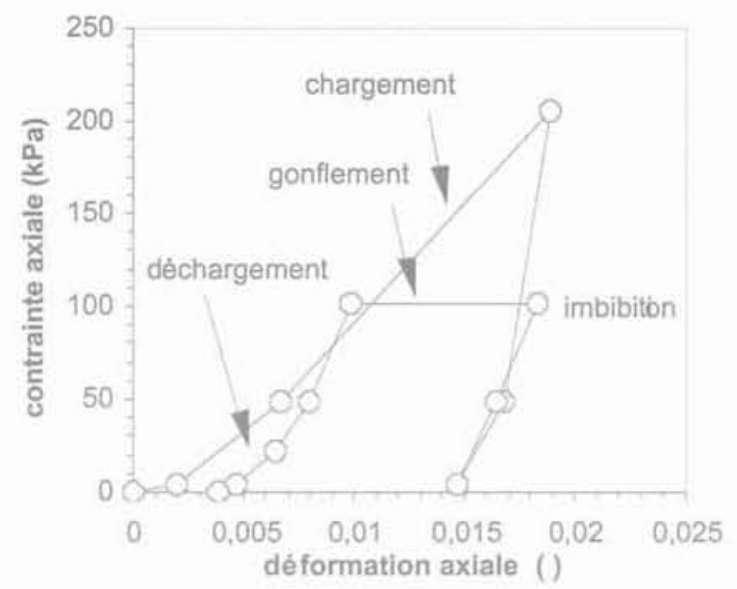

b. imbibition à $101 \mathrm{kPa}$

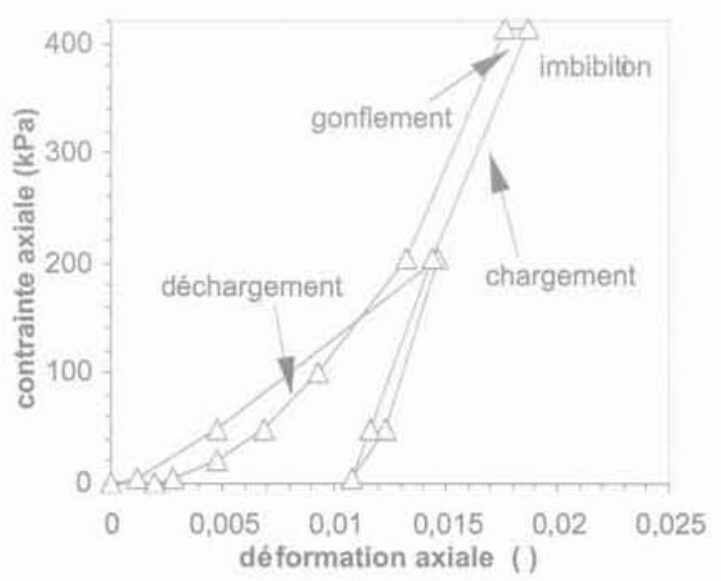

d. imbibition à $412 \mathrm{kPa}$

FIG. 12 Essais cedométriques en parallèle. Courbes contrainte déformation pour les différentes contraintes d'imbibition (Boutonnier et Serratrice, 2002).

Oedometer tests. Stress strain curves with wetting under different stresses (Boutonnier and Serratrice, 2002).

découpées dans l'échantillon E3 (12,20 - 13,70 m; èprouvettes TVS04, 05, 06 et 07). Elles sont placées dans leur ètat naturel à l'intérieur des cellules cedométriques sans eau. Un premier cycle de chargement déchargement est effectué par paliers jusqu'à la contrainte axiale $\sigma_{a \max }=200 \mathrm{kPa}$ en 24 heures. Puis les éprouvettes sont chargées par paliers sous les contraintes $\sigma_{\mathrm{a}}=48,101,202$ et $412 \mathrm{kPa}$ respectivement. Elles sont mises en imbibition $\left(\sigma_{\mathrm{a} \text { mbibitun }}\right)$ sous cette charge, puis elles sont déchargées par paliers de plusieurs jours, de 4 à 7 paliers respectivement, de façon à suivre le déchargement en détail. La durée totale des essais est de 500 heures.

\subsection{M.T.}

\section{Résultats obtenus}

Les résultats sont donnés sur les graphiques de la figure 12 avec les courbes déformation axiale contrainte axiale $\left(\varepsilon_{a^{\prime}} \sigma_{a}\right)$ pour chacune des éprouvettes. Les principales étapes des essais sont indiquées sur les courbes (chargement, imbibition, gonflement, déchargement).

La pression de gonflement est de l'ordre de $400 \mathrm{kPa}$ (gonflement quasi nul sous cette contrainte) et seules les éprouvettes ayant subj une imbibition sous des charges respectives de 48 et $101 \mathrm{kPa}$ présentent des gonflements significatifs.

Pour ces éprouvettes, il est possible de calculer une valeur de cy (tableau IV).

Compte tenu du gonflement observé et du mode opératoire décrit au $\$ 4.1$, les valeurs de $c_{y}$ mesurées correspondent au domaines D2.

\section{5}

\section{Comparaison $\Gamma$ estimé à partir des essais et $\Gamma$ estimé à partir des abaques}

L'équation (32) peut être présentée sous une autre forme en faisant apparaître le module œdométrique $E_{\text {codo. }}$. Elle peut également être combinée avec l'équation (36) ce qui donne:

$$
c_{v_{-} D 2}=\Gamma \cdot \frac{k_{v} \cdot E_{\text {ado }}^{\prime}}{\gamma_{w}}=\Gamma \cdot c_{v_{-} D 3 D 4 \_ \text {triax }} \cdot \frac{E_{\text {cedo }}^{\prime}}{K}
$$

Le rapport entre module œdométrique $\mathrm{E}_{\text {wato }}^{\prime}$ et module de compression volumique $\mathrm{K}$ peut-être estimé, dans le cas de l'élasticité linéaire isotrope, par: 
TAatEAUV Rapports entre module cdométrique et module de compression volumique sur trois sols naturels. Ratio between oedometer modulus and bulk modulus on three natural soils.

\begin{tabular}{|c|c|c|c|c|c|}
\hline Nature & $\begin{array}{l}E_{h}^{\prime} \\
(\mathrm{KPa})\end{array}$ & $\begin{array}{l}E_{y}^{*} \\
(\mathrm{KP} B)\end{array}$ & $v^{\prime}$ & $v^{*}$ hin & $\frac{E_{\text {matn }}}{K}$ \\
\hline $\begin{array}{l}\text { Argiles de Londres } \\
\text { Argiles du Lac Agassiz } \\
\text { Argiles de Romainville }\end{array}$ & $\begin{array}{l}22060 \\
9340 \\
24600\end{array}$ & $\begin{array}{l}11000 \\
4950 \\
22200\end{array}$ & $\begin{array}{r}0,19 \\
0,17 \\
0,4\end{array}$ & $\begin{array}{c}0 \\
0,23 \\
0,38\end{array}$ & $\begin{array}{l}1,9 \\
1,7 \\
1,6\end{array}$ \\
\hline
\end{tabular}

$$
\frac{E_{\text {cedo }}^{\prime}}{K}=3 \cdot \frac{1-v^{\prime}}{1+v^{\prime}}
$$

avec $v^{\prime}$ coefficient de Poisson.

Généralement. $v^{\prime}$ est compris entre 0,2 et 0,4

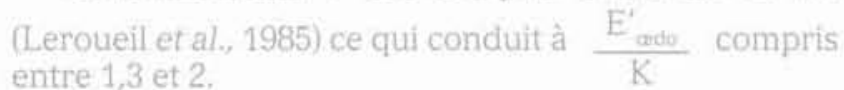

Dans le cas de sols présentant une élasticité orthotrope de révolution, l'équation (38) devient:

$$
\frac{E_{\text {oedo }}^{\prime}}{K}=\frac{\left(n-4 \cdot v_{v h}^{\prime}-2 \cdot v_{h h}^{\prime}+2\right) \cdot\left(1-v_{h h}^{\prime}\right)}{n-n \cdot v_{h h}^{i}-2 \cdot n^{2} \cdot v_{h h}^{\prime}}
$$

avec:

$\mathrm{n}=\mathrm{E}_{\mathrm{n}}^{\prime} / \mathrm{E}_{\mathrm{v}}^{\prime}$ rapport des modules horizontaux et verticaux;

$v_{v b}^{\prime}$ coefficient de Poisson dans la direction verticale sous une contrainte horizontale;

$v_{h h}^{\prime}$ coefficient de Poisson dans la direction horizontale sous une contrainte horizontale.

Le tableau $V \frac{E_{\text {mdo }}^{\prime}}{K}$ présente les valeurs de obtenues pour trois sols naturels surconsolidés (Mestat, 1993) ayant fait l'objet d'essais permettant de déterminer $\mathrm{n}, v_{\text {vi }}^{\prime}$ et $v_{\text {thi }}^{+}$

Comme avec l'élasticité isotrope, on trouve pour ces trois sols naturels surconsolidés que le rapport $\frac{E_{\text {tedo }}^{\prime}}{K}$ est compris entre 1,3 et 2.

Dans ces conditions, la valeur de $\Gamma$ peut être estimée en utilisant:

- l'équation (37):

- $\frac{E_{\text {cedo }}^{\prime}}{\mathrm{K}}$ compris entre 1,3 et 2;

- $c_{v-D 2}=1,6 \cdot 10^{-8} \mathrm{~m}^{2} / \mathrm{s}$ (valeur moyenne, $\mathrm{n}=9$ ):

- $c_{v_{\text {_tDas }} \text { _triax }}=1,310^{-8}$ et $1,910^{-8} \mathrm{~m}^{2} / \mathrm{s}$ (2 valeurs sous contraintes d'imbibition respectives de 48 et $101 \mathrm{kPa}$ ).
On obtient $0,4 \leq \Gamma \leq 1$.

S'agissant de mames silteuses, la succion d'entrée d'air est très probablement supérieure à $300 \mathrm{kPa}$ (Biarez et al., 1987). Dans ces conditions, les abaques (Figs, 6, 7 et 8) nous indiquent une valeur de $\Gamma$ comprise entre 0,4 et 1 qui est cohérente avec celle estimée expérimentalement.

Ces essais constituent donc une première validation, certes très partielle, de la théorie exposée dans les chapitres précédents.

\section{4}

\section{Conclusion}

Dans les sols fins proches de la saturation (avant la succion d'entrée d'air), la théorie présentée permet d'estimer de manière simple les tassements et gonflements différés à l'aide d'un couplage hydromécanique prenant en compte:

- la compressibilité du fluide interstitiel dans le domaine D3 (mélange eau + air avec succion négligeable):

- la succion dans le domaine D2 (domaine entre succion nulle et succion d'entrée d'air).

Cette théorie permet de montrer que les coefficients de consolidation verticale dans les domaines D2 (c. déterminé à partir d'un essai de gonflement libre à l'cedomètre) et D3 (cy déterminé à partir d'un essai œedométrique par palier) sont très souvent égaux et dans tous les cas du mème ordre de grandeur (pour un indice des vides donné).

L'analyse de certains essais réalisés pour la LGV EST permet d'obtenir une première validation partielle de la théorie.

Une validation plus franche de la théorie pourrait être obtenue avec des essais réalisés dans les domaines D2 et D3 sur le même échantillon à l'cedomètre (réalisation d'un essai de chargement par paliers après réalisation d'un essai de gonflement libre). 
Alonso E F Gens A Josa A - A constitutive model for partially saturated soils. Geotechnique 40,1990, p. 405-430.

Al-Mukhtar M. - Macroscopic behavior and microstructural properties of a kaolinite clay under controlled mechanical and hydraulic state. Proc. of the 1st Conference on Unsaturated Solls, Unsat95. Paris, 1. 1995, p. 3-9.

Atabek R.B., Félix B.. Robinet J.-C., Lahlou, R. - Rheological behaviour of saturated expansive clay materials. Workshop on stress Partitioning in Engineering Clay Barriers, Duke University, Durharn, NC. 1991.

Barden L. - Consolidation of compacted and unsaturated clays. Geotechnique 15 (3). 1965, p. 267-286.

Barden L. - Consolidation of clays copacted «dryp and "wet» of optimum water content. Geotechnique 24 (4). 1974, p. 605-625.

Biarez J., Fleureau, J.-M., Zerhouni M.- . Soepandii, B. S. - Variations de volume des sols argileux lors de cycles de drainage-humidification. Revue française de géotechnique 41, 1988, p. 63-71.

Black D.K., Lee K.L - Saturating laboratory samples by back pressure. J. Soil Mech. Found. End. ASCE 99, 1, 1973, p. 75-93.

Boutonnier L., Serratrice J.-F, - Caractérisation du comportement d'une marne en vue de la réalisation d'un déblai. Param 2002. Paris, Presses de l'ENPC/LCPC, 2002. p. 519-530.

Boutonnier L., Virollet M. - Tassements et gonflements instantanés dans les sols fins proches de la saturation. Revue française de géotechnique 104, 2003 . p. 3-19.

Brooks R.H. Corey A.T. - Hydraulic Properties of Porous Media. Colorado State Univ. Hydrol. Paper 3, 27, 1964, p. ??

Cafaro F., Cotecchia F. Cherubini C. Influence of structure and stress history on the drying behaviour of clays. Proc. Asian conference on unsafurated soils, Singapore, Balkema, 2000, p. 633-638.

Chang C.S., Duncan J.M. - Consolidation analysis for partly saturated clay by using an elastic-plastic effective stressstrain model. Int. J. for Numerical and Analytical Methods in Geomechanics 7. 1983, p. 39-55.

Chen Y.J. Yu P.J. - Pore pressure dissipation features of an unsaturated compacted soil. Proc. of the 1st Conference on Unsaturated Soils, Unsat'95, Paris, 2, 1995, p. 439-445

Delage, P. - Aspects du comportement des sols non saturés. Revue Francaise de géotechnique 40, 1987, p. 33-43.

Delage P., Cui Y.J. - L'eau dans les sols non saturés. C301. Traité Construction, C2, 2000.

Delage P., Fry J.-J. - Comportement des sols compactés : apport de la mécanique des sols non saturés. Revue française de géotechnique 92,2000 , p. 17-29.

Ed Diny S. Masrouri F. Tisot J.-P. - Détermination de la conductivité hydraulique d'un limon non saturé. Revue française de géotechnique 62, 1993. p. $67-74$

Fleureau J.M., Kheirbek-Saoud S., Soemitro R., Taibi S, - Behavior of clayey soils on drying-wetting paths. Canadian Geotechnical Journal 30 (2), 1993, p. $287-296$

Fleureau J.M., Verbrugge I.-C.. Huergo
P.J., Gomes Correia A. KheirbekSaoud S. - Aspects of the behavior of clavey soils on drying and wetting paths. Canadian Geotechnical Journal 39, 2002, p. $1341-1357$

Fourie A. B. Hofmann B.A. Mikula R.J. Lord R.F., Robertson P.K. - Partially saturated tallings sand below the phreatic surface. Geotechnique 51 (7) 2001, p. 577-585

Fredlund D.G. Morgenstern, N.R.--Stress state variables for unsaturated soils ASCE Journal of the Geotechnical Engineering Division 103 (5), 1977, p. 447 . 466

Fredlund D.G., Hasan J.U, - One-dimensional consolidation theory: unsaturated soils, Canadian Geotechnical Journal 16, 1979, p. 521-531.

Fredlund D.G., Rahardjo H. - Soils Mechanics for unsaturated soils. A WileyInterscience Publication, John Wiley \& Sons, 1993

Garcia-Bengochea I., Lovell C.W.. Altschaeffl A.G. - Relation between pore size distribution and permeability of silty clay. Journal of the Geotechnical Engineering Division ASCE 105 (GT7), 1979 , p. $839-856$

Geiser F. - Applicability of a general effective stress concept to unsaturated soils. Proc Unsaturated Soils for Asia, Rahardjo, Balkema 2000, p. 101-105.

Gens A., Alonso E.E. - A framework for the behaviour of unsaturated expansive clays. Canadian Geotechnical Journal 1 (29), 1992, p. 1013-1032.

Hilf J.W. - Estimating construction pore pressure in rolled earth dams. Proc, of $2^{\text {nd }}$ International Conference on Soit Mechanics and Foundation Engineering. Rotterdam, 3, 1948, p. 234-240.

Khalili N. - Application of the effective stress principle to volume change in unsaturated soils. Proc. Unsaturated Soils for Asia. Rahardjo, Balkerna 2000, p. $119-124$.

Khalili N. Khabbaz M.H. - On the theory of three-dimensional consolidation in unsaturated soils. Proceedings of the fifteenth international conference on soil mechanics and geotechnical engineering Istanbul 2, 1995, p. 745-750

Khalili N., Khabbaz, M.H. - A unique relationship for $x$ for the determination of the shear strength of unsaturated Soils. Géotechnique, 48, 1998, p. 681-688.

LeBihan J.-P., Leroueil. S. (2002). A mode) for gas and water flow through the core of earth dam. Canadian Geotechnical Journal 39, p. 90-102.

Ieroueil S. Magnan J.P. Tavenas F. Remblais sur argiles molles. Technique et Documentation, Lavoisier, Paris, 1985.

Li X.L., Collin F., Radu J-P., Bolle A., Charlier, R. - Experimental and numerical studies on the mechanical behaviour of two quasi saturated fine soils. Proc. of the fifteenth international conference on soil mechanics and geotechnical engi. neering. Istanbul 1, 2001, p. 597-600.

Lins A.H.P. Sandroni S.S. Sills G.C. - An experimental analysis of the development of pore-water pressure in an unsaturated compacted soil. Proc. of the 1st Conference on Unsaturated Soils Unsat' 95 , Paris, 2,1995 , p. $759-764$.

Lforet A. Alonso E.E. - Consolidation of unsaturated soils including swelling and collapse behavior. Geotechnique 30 (4), 1980, p. 449-477

Loret B. Khalili N. - A three phase model for unsaturated soils. Int. I. Numer. Anal. Meth. in Geomech. 24, 2000, p. 893-927.

Loret B., Khalili N. - An effective stress elastic-plastic model for unsaturated porous media. Mechanics of Materials 34. 2002, p.97-116.

Magnan J.P., Dang M.T. - Etude théorique et expérimentale de la compressibilité du fluide interstitiel dans un sol argileux prescue saturé. Bulletin de liaison des LPC. Paris, 1977, (n" spécial VIF), p. 129 137.

Magnan J.-P. Baghery S. Brucy M. Tavenas $E_{\text {. }}$ - Etude numérique de la consolidation unidimensionnelle en tenant compte des variations de la perméabilité et de la compressibilité du sol, du fluage et de la non-saturation. Bulletin de liaison des LPC 103, 1979, p. 83-94.

Magnan J,-P, Mieussens C, Soyez B., Vautrain J. - Essais codométriques. Méthodes d'essai LPC 13. Laboratoire central des Ponts et chaussées, 1985.

Mestat P. - Lois de comportement des qéomatériaux et modélisation par la méthode des éléments finis. Études et recherches LPC, série géotechnique GT 52, 1993.

Modaressi A., Abou-Bekr N., Fry J.-1, - Unified approach to model partially saturated and saturated soil. Proc. of the $8^{\text {m }}$ International Conference on Computer Methods and advances in Geotech. Morgantown, 1996, p. 1507-1513.

Murray E.J. - An equation of state for unsaturated solis. Canadian Geotechnical Journal 39, 2002, p. 125-140.

Nagaraj TS Miura N. - Soft Clay Behaviour, Analysis and Assessment. A.A. Balkema, Rotterdam, Brookfield, 2001.

Schuurman 1.E. - The compressibility of an air/water mixture and a theoretical relation between the air and water pressures. Geotechnique 16, 1966, p. 269-281.

Sills G.C. Thomas S.D, - Pore pressures in soils containing gas. Proc. of the workshop on chemo-mechanical coupling in clays; from nano-scale to engineering applications, Maratea, Italy, 28-30 june 2001. Di Maio, Hueckel \& Loret (eds), Balkema, 2001.

Sills G.C. Wheeler S.I. Thomas S.D. Gardner T.N. - Behaviour of offshore soils containing gas bubbles. Geotechnique 41 (2), 1991. p. 227-241.

Sridharan A., Altschaeff A.G., Diamond S.Pore size distribution studies. ASCE J. Solt Mech. Found. Div. 97, 1971, p.771-787.

Tavenas F., Jean P., Leblond P. Leroueil S. The permeability of natural clays. Part. II : permeability characteristics. Canadian Geotechnical Journal 20 (4), 1983. p. $645-660$

Thomas H.R., H.Y. - Modelling the behaviour of unsaturated soil using an elastoplastic constitutive model. Géotechnique 48 (2), 1998, p. 589-603.

Vaughan P.R. - Observations on the behaviour of clay fill containing occluded air bubbles. Geotechnique 53 (2), 2003, p. $265-272$.

Zerhouni M.1. Gérard C. Fleureau J.M. Étude du retrait de deux sols argileux naturels. Actes du colloque Magi'50, Vandceuvre, 1998, p. 15-20. 